\title{
G

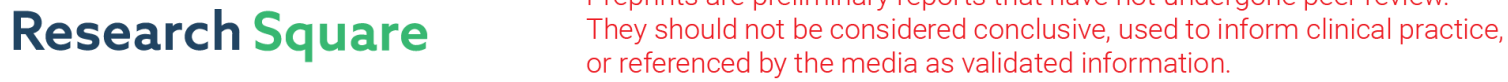 \\ Inhibition of BACE1 Facilitates Macrophage-based Immunotherapy to Suppress Malignant Growth of Glioblastoma
}

\section{Kui Zhai}

Department of Cancer Biology, Lerner Research Institute, Cleveland Clinic, Cleveland, OH 44195

\section{Zhi Huang}

Cleveland Clinic

\section{Weiwei Tao}

Cleveland Clinic

\section{Xiaoguang Fang}

Cleveland Clinic

\section{Qian Huang}

Cleveland Clinic

\section{Xiaoxia Li}

Cleveland Clinic https://orcid.org/0000-0002-4872-9525

\section{George Stark}

Cleveland Clinic

Thomas Hamilton

Cleveland Clinic

\section{Shideng Bao ( $\sim$ baos@ccf.org )}

Lerner Research Institute, Cleveland Clinic https://orcid.org/0000-0002-4236-2662

\section{Article}

Keywords: Tumor-associated macrophages, Glioblastoma, BACE1, MK-8931, Immunotherapy, Phagocytosis, Irradiation, IL-6/sIL-6R/STAT3

Posted Date: September 4th, 2020

DOI: https://doi.org/10.21203/rs.3.rs-61939/v1

License: (1) (1) This work is licensed under a Creative Commons Attribution 4.0 International License. Read Full License 
Version of Record: A version of this preprint was published at Nature Cancer on November 8th, 2021. See the published version at https://doi.org/10.1038/s43018-021-00267-9. 


\section{Abstract}

Malignant tumors, including glioblastoma (GBM), contain abundant tumor-associated macrophages (TAMs) that mainly promote tumor growth and therapeutic resistance. Reprograming tumor-promoting TAMs (pTAMs) into tumor-suppressive TAMs (sTAMs) represents an attractive therapeutic strategy. We discovered that inhibition of the $\beta$-site amyloid precursor protein cleaving enzyme 1 (BACE1) by MK-8931 potently redirects pTAMs into STAMs and promotes macrophage phagocytosis of glioma cells to suppress the malignant growth of GBM. Moreover, low doses of radiation markedly enhance TAM infiltration and synergize with MK-8931 treatment. BACE1 is preferentially expressed by pTAMs in human GBMs and is required for maintaining PTAM polarization through trans-IL-6/sIL-6R/STAT3 signaling. As several BACE1 inhibitors, including MK-8931, previously developed for Alzheimer's disease, were shown in clinical trials to be safe for humans, repurposing these inhibitors for cancer therapy should be straightforward. Collectively, this study offers a promising therapeutic approach, through inhibition of BACE1, to facilitate the macrophage-based tumor immunotherapy.

\section{Highlights}

- BACE1 inhibition by MK-8931 promotes TAM phagocytosis to eliminate glioma cells.

- Disrupting BACE1 redirects pTAMs into sTAMs to suppress GBM tumor growth.

- Low doses of radiation enhance TAM infiltration and synergize with MK-8931 treatment.

- BACE1 maintains pTAMs via trans-IL-6/sIL-6R/STAT3 signaling.

- High levels of BACE1 predict poor prognosis for GBM patients.

\section{Introduction}

Tumor-associated macrophages (TAMs) are critical immune cells within the tumor microenvironment $(\mathrm{TME})^{1,2}$, and their abundance is often associated with poor prognosis in most malignant tumors, including glioblastoma (GBM) ${ }^{3,4}$. TAMs can be functionally categorized into tumor-promoting (pTAMs) and tumor-suppressive (sTAMs) types, although each type includes several subpopulations, as more than a dozen TAM subsets have been recently identified by single-cell RNA sequencing ${ }^{1,5,6}$. The majority of TAMs in the TME are PTAMs, which support malignant growth and promote therapeutic resistance ${ }^{1,2,7,8}$. pTAMs exhibit an M2 macrophage-like phenotype ${ }^{2,5,9}$ with expression of markers including CD163, arginase-1 (ARG1), and FIZZ12,10-13. In contrast, sTAMs display properties of M1-like macrophages ${ }^{2}$ and express M1-specific markers such as HLA-DR, inducible nitric oxide synthase (iNOS), and CD11 $c^{2,10-13}$. Recent studies indicate that pTAMs play immunosuppressive roles in the $\mathrm{TME}^{14}$ and have a negative impact on therapy with immune checkpoint inhibitors ${ }^{15}$. Therefore, reprograming pTAMs into sTAMs may not only directly suppress malignant growth but may also offer the opportunity to improve current $T$ cellbased immunotherapy. The goal of our study is to develop macrophage-based immunotherapy for treating malignant tumors, including GBMs, that contain abundant $\mathrm{TAMs}^{16}$. 
GBM is the most common and fatal brain cancer ${ }^{17}$ highly resistant to therapies including current antiPD1 immunotherapy ${ }^{18,19}$. GBM inevitably recurs after surgical resection and radio-chemotherapy ${ }^{20,21}$. We and the others have demonstrated that pTAMs, glioma stem cells (GSCs), and the interplay between them play vital roles in promoting tumor progression and therapeutic resistance in $\mathrm{GBMs}^{7,22,23}$. TAMs and GSCs are often located in the perivascular niche in $\mathrm{GBM}^{10,24}$ and actively interact at cellular and molecular levels to support malignant growth ${ }^{10-12,25}$. As the majority of TAMs in a GBM are PTAMs, which usually lose the ability to phagocytize tumor cells ${ }^{26}$, we hypothesized that redirecting pTAMs into sTAMs to activate macrophage phagocytosis against glioma cells, including GSCs, might suppress malignant tumor growth to effectively improve GBM treatment. To discover small molecules that can potently activate TAM phagocytosis against glioma cells, we designed a cell-based screen, using human iPS cells (iPSC)-derived macrophages and glioma cells including GSCs to identify drug candidates and their potential molecular targets on TAMs. We identified MK-8931, a specific inhibitor of BACE1 ( $\beta$-site amyloid precursor protein cleaving enzyme 1), as a top candidate to promote macrophage phagocytosis to eliminate cancer cells, and thus defined BACE1 as a potential therapeutic target to reprogram pTAMs into sTAMs for developing the macrophage-based tumor immunotherapy.

BACE1 is a trans-membrane aspartyl protease that is responsible for the production of amyloid beta peptide $(A B)$ in the brains of patients with Alzheimer's disease $(A D)^{27-30}$. Since its discovery, BACE1 has been widely investigated as a therapeutic target for $A D^{31}$, and several BACE1 inhibitors, including MK8931, have been tested in clinical trials for AD treatment ${ }^{32}$. In this study, we found that BACE1 is preferentially expressed by PTAMs and that BACE1-mediated trans-IL-6/sIL-6R/STAT3 signaling is required for the maintenance of pTAMs. We demonstrate that targeting BACE1 with the specific inhibitor, MK-8931, effectively converts pTAMs into sTAMs and promotes TAM phagocytosis against glioma cells in vitro and in vivo. Importantly, our preclinical studies demonstrate that BACE1 inhibition by MK-8931 potently suppresses tumor growth of human GBM in xenograft models, suggesting that pharmacological targeting of BACE1 can be used to activate TAMs to exert anti-tumor activity. Furthermore, we found that low doses of radiation markedly induce the infiltration of TAMs and synergize with MK-8931 to suppress tumor growth. Thus, we used low-dose radiation to enhance the infiltration of TAMs into tumors that contain relatively few TAMs and then treat the tumors with MK-8931 to activate TAM phagocytosis to improve the anti-tumor efficacy. Our studies indicate that targeting BACE1 with MK-8931, alone or in combination with low-dose radiation, is a promising therapeutic approach for treating malignant cancers, including glioblastoma.

MK-8931 (Verubecestat), a non-peptidic class of BACE1 inhibitor originally developed for AD treatment ${ }^{33}$, penetrates the blood-brain barrier (BBB) very well and blocks BACE1 activity efficiently in the brain ${ }^{34}$. MK8931 is the first BACE1 inhibitor that has proceeded to a phase III clinical trial for AD patients ${ }^{31}$. Recent clinical studies demonstrated that MK-8931 is generally safe and well tolerated in healthy adults ${ }^{35,36}$ and $A D$ patients ${ }^{34,37,38}$. Although targeting BACE1 by MK-8931 is not effective for treating AD patients ${ }^{37,38}$, our study strongly indicates that this drug can be repurposed for tumor immunotherapy as it can 
effectively redirect pTAMs into sTAMs to eliminate cancer cells in GBM tumors. Thus, this study offers a macrophage-based immunotherapy through BACE1 inhibition with a small molecule that promotes TAM phagocytosis of cancer cells to suppress malignant growth, which may have great potential to improve the treatment of lethal cancers.

\section{Results}

\section{Identification of the BACE1 Inhibitor MK-8931 as a Potent Activator of Macrophage Phagocytosis of Glioma Cells}

To identify small molecular modulators that promote phagocytosis of cancer cells by macrophages, we developed a fluorescent phagocytosis assay, using GFP-labeled human iPSC-derived macrophages (in green) and tdTomato-expressing human glioma stem cells (GSCs, in red) (Fig. 1a). This assay detects phagocytosis as fluorescent inclusion bodies derived from GSCs $\left(\right.$ tdTomato $^{+}$) within macrophages $\left(\mathrm{GFP}^{+}\right)^{39}$. To obtain GFP-expressing macrophages, we transduced iPSCs with a GFP expression construct,

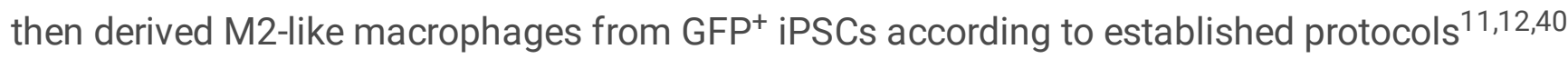
(Supplementary Fig. 1a,b). We confirmed that GFP was constitutively expressed during macrophage differentiation and that the iPSC-derived macrophages expressed total macrophage markers, including CD11b and IBA1, and M2 markers, including ARG1 and FIZZ1 (Supplementary Fig. 1c). Thus, small molecules that activate macrophages $\left(\mathrm{GFP}^{+}\right)$to engulf GSCs (tdTomato ${ }^{+}$) can be detected with a fluorescent microscope (Fig. 1a). We initially screened an inhibitor library (SelleckChem) and some known drugs that displayed excellent BBB permeability and low toxicity in phase II/III clinical trials for other diseases, including AD. We obtained seven "hits" and further identified five BACE1 inhibitors, including MK-8931 as the most promising candidates. The screening assay showed that MK-8931 treatment promoted phagocytosis of the iPSC-derived macrophages against GSCs (Fig. 1b,c). Moreover, MK-8931 treatment also augmented phagocytosis of bone marrow-derived macrophages (BMDMs) against GSCs (Fig. 1d,e), suggesting that MK-8931 is a potent activator of macrophage phagocytosis of cancer cells. As MK-8931 was originally developed as a BACE1-specific inhibitor for AD patients ${ }^{33,41}$, we then confirmed that BACE1 was expressed by the iPSC-derived macrophages but not by the iPSCs (Fig. 1f). These data indicate that BACE1 inhibition by MK-8931 promotes macrophage phagocytosis of glioma cells in vitro.

We next sought to determine whether BACE1 inhibition by MK-8931 promotes TAM phagocytosis in vivo in GSC-derived GBM xenografts that contain abundant TAMs ${ }^{10-12}$. We initially examined the expression of BACE1 in TAMs in GBM xenografts derived from human GSCs (CCF-3264 or CCF-DI315) by double immunofluorescent staining, finding that BACE1 was co-expressed usually with the total TAM markers CD11b and IBA1 and the PTAM markers CD163 and FIZZ1, but rarely with the sTAM markers HLA-DR and CD11c (Supplementary Fig. 2), suggesting that BACE1 is mainly expressed by pTAMs in the GBM xenografts. We then treated mice bearing intracranial GBM xenografts with MK-8931 (30 mg/kg) or the vehicle control once daily by oral gavage for two weeks and harvested the tumors to analyze the effect of MK-8931 treatment on macrophage phagocytosis. To detect TAM phagocytosis in tumor sections of 
GBM xenografts, we labeled the TAMs with anti-IBA1 antibody (in green) and the human glioma cells with antibody against the human-specific antigen TRA-1-85 (in red), finding that MK-8931 treatment resulted in a significant increase of inclusion bodies derived from the glioma cells (red) within TAMs (green) (Fig. 1g,h), indicating that BACE1 inhibition by MK-8931 activates TAMs to engulf glioma cells in GBM tumors. Collectively, these data indicate that BACE1 inhibition by MK-8931 promotes macrophage phagocytosis of glioma cells in vitro and in vivo.

\section{MK-8931 Treatment Potently Inhibits Tumor Growth and Extends the Survival of Mice Bearing GBM Xenografts}

We next investigated whether MK-8931 treatment could suppress GBM growth and impact survival. To address this important point, we treated mice bearing GBM xenografts derived from the luciferaseexpressing human GSCs (CCF-3264 or CCF-DI315) with MK-8931 (30 mg/kg/daily) or a vehicle control by oral gavage and then monitored tumor growth by using the In Vivo Imaging System (IVIS) (Fig. 2a). Bioluminescent imaging demonstrated that MK-8931 treatment potently inhibited GBM tumor growth (Fig. 2b,c). MK-8931 treatment also substantially extended the survival of mice bearing GBM tumors (Fig. 2d). To further understand the cellular effects of MK-8931 treatment on GBM tumor growth, we examined TAM phagocytosis, tumor angiogenesis, cell apoptosis, and proliferation in the MK-8931treated and the control tumors. MK-8931 treatment not only promoted TAM phagocytosis (Fig. 1g,h) but also increased cell apoptosis as indicated by cleaved caspase 3 staining (Supplementary Fig. 3a,b), reduced vessel $\left(\mathrm{CD} 31^{+}\right)$density (Supplementary Fig. 3c,d), decreased cell proliferation (Ki67 $)$ (Supplementary Fig. 3e,f), and reduced GSC population (SOX2+) in the tumors (Supplementary Fig. 3g,h). These cellular alterations induced by MK-8931 treatment in the GBM xenografts were unlikely to be the direct effects of MK-8931 on glioma cells, including GSCs, because MK-8931 in cell culture did not affect proliferation, sphere formation, cell viability, or apoptosis of GSCs or non-stem glioma cells in vitro (Supplementary Fig. 4). Collectively, our preclinical data demonstrate that targeting BACE1 by MK-8931 potently suppresses malignant growth of GBMs. Moreover, these data suggest that BACE1 inhibition by MK-8931 not only promotes TAM phagocytosis to eliminate glioma cells but also modulates the tumor microenvironment and thus potently inhibits tumor growth.

\section{BACE1 Is Required for the pTAM Maintenance and Controls the TAM Phenotype Switch}

Because the cellular alterations induced by MK-8931 treatment are similar to those defined in tumors when PTAMs are inhibited ${ }^{10-12,42}$, we were prompted to interrogate whether BACE1 plays a functional role in the maintenance of pTAMs. We generated pTAM-like M2 macrophages from the PMA-primed U973 cells (called M0 macrophages) by using the cytokines IL4, IL10 and TGF- $\beta$ according to an established protocol ${ }^{11,12}$ (Supplementary Fig. 5a), and found that the pTAM-like M2 macrophages derived from U937 monocytes expressed high levels of BACE1 at both the mRNA and protein levels, as measured by RT-PCR and immunoblot analyses (Supplementary Fig. 5b,c). We then examined the effects of BACE1 disruption by shRNA on PTAM-like macrophages, showing that BACE1 knockdown markedly reduced the expression of the pTAM markers CD163 and ARG1, while expression of the total TAM marker IBA1 was not affected 
(Supplementary Fig. 5d). This result was further confirmed by using the BACE1 inhibitor MK-8931 (Supplementary Fig. 5e). As BACE1 disruption or inhibition did not impact the expression of the total TAM marker IBA1 (Supplementary Fig. 5d,e), we speculated that targeting BACE1 might promote the phenotypic transition of PTAMs into sTAMs. To address this possibility, we knocked BACE1 down in PTAM-like M2 macrophages derived from U937 monocytes, finding that BACE1 disruption indeed induced the expression of sTAM markers, including HLA-DR and iNOS (Fig. 3a), while expression of the pTAM markers CD163 and ARG1 was dramatically reduced after BACE1 disruption (Fig. 3a and Supplementary Fig. 5d). Moreover, functional inhibition of BACE1 by MK-8931 in pTAM-like macrophages effectively induced expression of the sTAM markers HLA-DR and iNOS, but inhibited expression of the pTAM markers CD163 and ARG1 in a dose- and time-dependent manner (Fig. 3b,c). These data indicate that BACE1 disruption or inhibition redirects pTAMs into sTAMs in vitro.

Next we studied the role of BACE1 in the TAM phenotype switch in vivo by examining pTAM and STAM populations in control or MK-8931-treated GBM xenografts. MK-8931 treatment strongly reduced the density of pTAMs, as marked by $\mathrm{CD} 163^{+} / \mathrm{IBA} 1^{+}$or $\mathrm{FIZZ1} 1^{+} / \mathrm{IBA} 1^{+}$cells (Fig. $3 \mathrm{~d}$,e and Supplementary Fig. $6 \mathrm{a}, \mathrm{b})$. In contrast, the density of sTAMs, marked by HLA-DR ${ }^{+} / \mathrm{IBA} 1^{+}$or $\mathrm{CD} 11 \mathrm{c}^{+} / \mathrm{IBA} 1^{+}$cells, was strikingly increased in tumors treated with MK-8931, relative to controls (Fig. 3f,g and Supplementary Fig. $6 c, d)$. These data suggest that BACE1 inhibition can reprogram pTAMs into sTAMs in vivo. To further address whether this effect of BACE1 disruption functionally impacts tumor growth, we established a GBM xenograft model by co-transplanting monocyte-derived pTAM-like M2 macrophages expressing shBACE1 (BACE1 shRNA) or shNT (non-targeting shRNA) with GSCs expressing luciferase, as illustrated in Supplementary Fig. 7a. Co-transplantation of GSCs with monocyte-derived pTAM-like macrophages expressing shNT substantially promoted GBM tumor growth (Supplementary Fig. 7b,c) and reduced the survival of mice relative to control mice implanted with GSCs alone (Supplementary Fig. 7d), consistent with a tumor-promoting role of pTAMs $^{11}$. In contrast, co-transplantation of GSCs with monocyte-derived pTAM-like macrophages expressing shBACE1 (disrupting BACE1) did not augment GBM tumor growth, but rather suppressed malignant growth relative to GSCs alone (Supplementary Fig. 7b,c). Consequently, the survival of mice implanted with GSCs and the pTAM-like macrophages expressing shBACE1 was extended relative to mice implanted with GSCs alone or GSCs plus monocyte-derived pTAM-like macrophages expressing shNT (Supplementary Fig. 7d), indicating that BACE1 disruption in TAMs functionally reprograms PTAMs into sTAMs, resulting in anti-tumor activity. Collectively, these data indicate that BACE1 is required for maintaining PTAMs, and that BACE1 inhibition results in phenotypic switch of pTAMs into sTAMs, leading to suppression of tumor growth, and highlighting the role of BACE1 as a critical modulator in regulating TAM function in malignant tumors.

\section{BACE1 Maintains pTAMs by Catalyzing the Shedding of IL-6R and Activating Trans-IL-6/sIL-6R/STAT3 Signaling}

Since BACE1 is required for maintaining pTAMs, we next sought to understand the molecular mechanisms by which it drives pTAM polarization. Because STAT3 and STAT6 are key transcriptional regulators in M2 macrophage polarization ${ }^{43,44}$, we interrogated their potential role in the BACE1-mediated 
maintenance of pTAMs. Surprisingly, we found that the activating phosphorylation of STAT3 (pSTAT3Y705) but not STAT6 (pSTAT6-Y641) was substantially down-regulated after BACE1 disruption by shBACE1 (Fig. 4a) or inhibition by MK-8931 (Fig. 4b). Consistently, MK-8931 treatment in vivo profoundly reduced the number of pSTAT3 $^{+}$TAMs (identified as pSTAT3 ${ }^{+} /$IBA $^{+}$) and total pSTAT3 ${ }^{+}$cells in GBM xenografts (Fig. 4c-e). To further determine whether BACE1 functions through STAT3 signaling to maintain pTAMs, we examined whether ectopic expression of a constitutively active STAT3 (STAT3-C) could rescue the attenuated PTAM phenotype induced by BACE1 disruption or inhibition. Indeed, ectopic expression of STAT3-C restored the expression of the PTAM markers CD163 and ARG1 in monocytederived macrophages (Fig. 4f,g). Importantly, ectopic expression of STAT3-C abolished the increased expression of the sTAM markers iNOS and HLA-DR that was induced by BACE1 disruption or inhibition in the macrophages (Fig. 4f,g). Thus, ectopic expression of STAT3-C restored the pTAM phenotype impaired by BACE1 disruption or inhibition. Collectively, these data demonstrate that BACE1 maintains the pTAMs mainly through STAT3 activation, indicating that BACE1-mediated STAT3 activation is required for the pTAM maintenance.

To understand how BACE1 regulates STAT3 activation in PTAMs, we analyzed potential substrates of BACE1 (Supplementary Fig. 8a), finding that ten of them are involved in the regulation of STAT3 activity and eight are reported to play a role in macrophage polarization (Supplementary Fig. 8b). We further investigated four substrates, including the IL- 6 receptor (IL-6R) that functionally overlap in regulating STAT3 activity and macrophage polarization. To this end, we found that BACE1 mediates the shedding of IL-6R by functioning as a transmembrane protease, as BACE1 disruption or inhibition reduced not only the amount of the N-terminal fragment of IL-6R (called the soluble IL-6R, or sIL-6R) in the conditioned media of macrophages (Fig. 4h,i, top panels) but also reduced the membrane-bound C-terminal fragments of IL-6R (CTF) in macrophage lysates (Fig. 4h,i), while the amount of full-length IL-6R (FL) was increased in lysates of macrophages upon BACE1 disruption or inhibition (Fig. 4h,i). These data indicate that the sIL-6R released into the extracellular space is generated by the BACE1-mediated cleavage of membrane-bound full length IL-6R. It is well known that sIL-6R binds to IL- 6 and forms an IL-6/sIL-6R complex that retains the capacity to bind to glycoprotein 130 (gp130) and thus to activate STAT3 signaling ${ }^{45}$. This signaling mechanism, termed the trans-IL-6/sIL-6R/STAT3 pathway, plays a key role in the tumor microenvironment, driving tumor growth ${ }^{46-48}$. Importantly, IL-6/STAT3 activation induces the polarization of pTAMs and promotes cancer progression ${ }^{49,50}$. In fact, approximately $70 \%$ of the secreted IL-6 binds to sIL-6R in the circulation ${ }^{51}$. Thus, sIL-6R, functioning as a carrier of IL-6, is able to prolong the half-life of IL- 6 in vivo and to stabilize IL- 6 signaling ${ }^{52}$, resulting in a dramatic increase of STAT3 activation ${ }^{53}$. Consistently, we demonstrate that sIL-6R enhances IL-6-induced STAT3 activation in macrophages (Fig. 4j). Collectively, our data indicate that BACE1-mediated shedding of full length IL-6R generates sIL-6R, which promotes STAT3 activation induced by IL-6 to maintain the polarization of pTAMs (Fig. 4k).

Low Doses of Radiation Enhance TAM Infiltration and Effectively Synergize with MK-8931 Treatment to Inhibit Malignant Growth 
Our preclinical study has demonstrated that targeting BACE1 can effectively reprogram pTAMs into sTAMs to suppress GBM tumor growth. To improve the efficacy of macrophage-based immunotherapy through BACE1 inhibition for some tumors that may contain relatively few TAMs, we sought to find an effective way to enhance the infiltration of macrophages into tumors. Because low-dose irradiation (IR) promotes macrophage infiltration into tumors ${ }^{54}$, we speculated that IR-enhanced TAM infiltration could synergize with MK-8931 treatment to activate macrophage phagocytosis and convert pTAMs into sTAMs more effectively. To test this hypothesis, we irradiated mouse brains bearing GSC-derived xenografts with a low dose of IR $(2 \times 2 \mathrm{~Gy})$ to allow infiltration of more TAMs into the tumors and then treated the mice with MK-8931 (Fig. 5a). The combined treatment achieved the strongest inhibition of GBM tumor growth, relative to treatment with IR or MK-8931 alone (Fig. 5b,c). As a consequence, the combined treatment conferred the longest survival extension among four groups of mice (Fig. 5d). To determine the properties and phenotypes of TAMs in the GBM xenografts treated with IR, MK-8931, or IR plus MK-8931, we examined TAM density and subtypes in tumors from four groups of mice with different treatments. Total TAM density (IBA $1^{+}$cells) was remarkably increased by treatment with a low dose of IR (Supplementary Fig. 9a-c), consistent with previous reports that IR enhances TAM infiltration into tumors ${ }^{54,55}$. Surprisingly, the majority of TAMs induced by the low dose of IR were pTAMs but not sTAMs, as shown by the expression of CD163 but not HLA-DR (Supplementary Fig. 9a-c). However, MK-8931 treatment effectively converted these pTAMs $\left(\mathrm{CD} 163^{+}\right)$into sTAMs $\left(\mathrm{HLA}-\mathrm{DR}^{+}\right)$in the GBM xenografts treated with IR plus MK8931 (Supplementary Fig. 9a-c), suggesting that BACE1 inhibition by MK-8931 is able to redirect IRenhanced pTAMs into tumor-suppressive macrophages. Moreover, immunostaining analyses of the apoptotic marker cleaved caspase 3 in the GBM tumors demonstrated that the combined treatment resulted in significantly more apoptotic cell death than treatments with MK-8931 or IR alone (Supplementary Fig. 9d,e). Importantly, the apoptosis induced by the combined treatment $(25.9 \pm 3.1 \%)$ was notably greater than the sum of apoptosis induced by MK-8931 alone $(10.4 \pm 0.9 \%)$ or IR alone (3.1 \pm $0.7 \%$ ) (Supplementary Fig. 9e). Collectively, these data demonstrate that the enhanced TAM infiltration stimulated by a low dose of IR effectively synergizes with MK-8931 to suppress malignant growth and thus increases the survival of the tumor-bearing animals, highlighting the promising therapeutic potential of the macrophage-based tumor therapy using MK-8931 in combination with a low dose of IR.

\section{BACE1 Is Highly Expressed by pTAMs in human GBMs and Predicts Poor Prognosis}

To interrogate the clinical significance of BACE1 in human GBMs, we examined its expression in human GBM surgical specimens by double immunofluorescent staining, finding that BACE1 was detected in the fraction of total TAMs marked by IBA1 or CD11b (Fig. 6a). Quantitative analysis indicated that approximately $60 \%$ of the IBA $1^{+}$TAMs and about $67 \%$ of the CD $11 \mathrm{~b}^{+}$TAMs express BACE1 (Fig. $6 \mathrm{~b}$ ). Further examination demonstrated that BACE1 was mainly co-expressed with the pTAM marker CD163 or FIZZ1 (Fig. 6C). Quantification showed that the majority of pTAMs express BACE1, as approximately 83\% of the $\mathrm{CD} 163^{+}$TAMs and $86 \%$ of the FIZZ1 ${ }^{+}$TAMs are BACE1-positive (Fig. 6d). In contrast, BACE1 was rarely co-expressed with the sTAM marker HLA-DR or CD11c (Fig. 6e,f). The preferential expression of BACE1 in pTAMs was consistently found in all human GBM specimens (12 cases) examined. To further 
investigate the clinical relevance of BACE1 expression in PTAMs in human GBM tumors, we analyzed the relationship between BACE1 expression and the survival of GBM patients in several databases, including the Cancer Genome Atlas (TCGA), Rembrandt, Gravendeel and LeeY, finding an inverse correlation between BACE1 expression and patient survival in all these databases (Supplementary Fig. 10a-d). GBM patients with higher BACE1 expression levels in their tumors clearly had a worse survival (Supplementary Fig. 10a-d), indicating that BACE1 expression predicts poor prognosis. The inverse correlation between BACE1 expression in PTAMs and the prognosis is consistent with the fact that pTAMs support tumor growth and malignant progression in human GBMs. Collectively, these data demonstrate that BACE1 is preferentially expressed by pTAMs in most human GBM tumors and predicts a poor prognosis for these patients, validating the therapeutic promise of targeting BACE1 by an inhibitor such as MK-8931.

\section{Discussion}

TAMs are the most abundant immune cells in malignant tumors, including GBMs ${ }^{1,2}$. Most TAMs play supportive roles to promote tumor growth and malignant progression ${ }^{8}$ including invasion ${ }^{56}$, immune evasion $^{57}$, and therapeutic resistance ${ }^{7}$. Thus, either targeting tumor-promoting TAMs (pTAMs) or reprograming pTAMs into tumor-suppressive macrophages (sTAMs), to activate their phagocytosis against cancer cells, is an attractive therapeutic approach. Targeting or reeducating TAMs by inhibiting colony-stimulating factor 1 receptor (CSF-1R) showed suppression of GBM growth in mouse models ${ }^{42}$, but clinical trials with CSF-1R inhibitors failed to show significant efficacy for GBM patients ${ }^{58}$. Because CSF-1R is also expressed on circulating monocytes and other normal cells, targeting it resulted in serious toxic effects in the clinical trials ${ }^{59}$, making therapeutics targeting CSF-1R ineffective for clinical use ${ }^{58,60}$. In addition, CD47 inhibition alone modestly inhibits GBM progression ${ }^{61}$ but leads to therapeutic resistance in other cancer models ${ }^{62}$. In this preclinical study, we demonstrate that reprograming pTAMs into sTAMs by inhibiting BACE1 with the specific inhibitor MK-8931 potently promotes TAM phagocytosis against glioma cells and effectively inhibits tumor growth, extending the survival of mice bearing GBM tumor (Fig. 7, graphic abstract). As BACE1 inhibition by MK-8931 has been demonstrated to be safe in healthy adults ${ }^{35,36}$ and to be well-tolerated by $A D$ patients ${ }^{37,38}$, and since MK-8931 can penetrate the BBB very well ${ }^{34}$, redirecting pTAMs into sTAMs by BACE1 inhibition with MK-8931 may overcome the shortcomings of targeting CSF-1R and offer a macrophage-based promising immunotherapy to improve treatment of malignant tumors, including GBM.

BACE1 is a type I transmembrane $\beta$-secretase that plays important roles in physiological and pathophysiological processes ${ }^{63,64}$. BACE1 cleaves amyloid precursor protein to cause accumulation of $A \beta$ production in the brains of $A D$ patients ${ }^{27-30}$. However, BACE1 deficiency is well-tolerated in knockout mice, without obvious effects on development, behavior, or fertility ${ }^{63}$, indicating that targeting BACE1 should not result in side effects. Interestingly, microglia in BACE1-deficient mice also increased phagocytosis toward cellular debris after nerve damage ${ }^{65,66}$. However, to the best of our knowledge, the functional significance of BACE1 in regulating TAMs has not been reported. In this study, we 
demonstrated that BACE1 plays a critical role in maintaining PTAMs in tumors. BACE1 inhibition potently converts pTAMs into sTAMs to promote TAM phagocytosis against cancer cells in vivo. Importantly, BACE1 inhibition did not cause the death of pTAMs but redirected them into sTAMs that have tumorsuppressive roles. Thus, targeting BACE1 represents an attractive therapeutic strategy to improve tumor immunotherapy.

Our preclinical studies indicate that MK-8931 is a promising drug to modulate TAM function for reclaiming anti-tumor activities of macrophages in tumors. To enhance the therapeutic efficacy of MK8931, we found that a low dose of irradiation (IR) remarkably augmented TAM infiltration and effectively synergized with MK-8931. Radiotherapy is a standard treatment for $\mathrm{GBM}^{17}$, but this tumor is highly resistant to IR, partially due to the population of GSCs that resist to conventional treatments and contribute to tumor recurrence ${ }^{22,23}$. In addition, IR often triggers an inflammation response and remodulate the tumor microenvironment to induce therapeutic resistance ${ }^{67,68}$. Our study demonstrates that a low dose of IR markedly enhances TAM infiltration into GBM tumors. Surprisingly, the increased TAMs induced by the IR in tumors were mainly pTAMs, although a previous in vitro study showed that IR can induce the polarization of human and murine monocytes toward M1-like macrophages ${ }^{69}$. It is possible that IR affects macrophages in vitro and in vivo in a different manner and that low and high doses of IR differentially impact macrophage polarization. Interestingly, another study showed that IR could induce BACE1 expression ${ }^{70}$, which should promote the maintenance of pTAMs. Although treatment with a low dose of IR alone does not significantly impact tumor growth, it provides a powerful tool to enhance TAM infiltration into tumors, which allows MK-8931 treatment to redirect the increased pTAMs into more sTAMs to better suppress tumor growth. This therapeutic strategy is particularly important for those solid tumors that contain relatively few TAMs. Most malignant tumors contain abundant TAMs and treatment with MK-8931 alone should effectively promote TAM phagocytosis to engulf cancer cells, but certain types of tumors in some patients may have fewer TAMs. In either situation, the combination of MK-8931 with a low dose of IR should enhance therapeutic efficacy. Because some tumors may not contain enough infiltrating $T$ cells to facilitate current immunotherapy with immune checkpoint inhibitors such as anti-PD1 antibody, the combination of low dose of IR with MK-8931 may provide an alternative strategy to overcome the poor response of some malignant tumors to current immunotherapy.

The molecular mechanisms underlying the polarization and maintenance of TAMs were poorly understood. In this study, we found that BACE1 mediates the critical shedding of the membrane-bound full length IL-6R by functioning as a transmembrane protease to generate the soluble form of IL-6R (sIL$6 \mathrm{R})$, which activates the trans-IL-6/sIL-6R/STAT3 signaling to maintain the polarization of pTAMs (Fig. 4k). Our in vivo study further demonstrated that inhibiting BACE1 with MK-8931 suppressed STAT3 activation in TAMs, resulting in reduced number of pTAMs and increased density of sTAMs, as well as enhanced macrophage phagocytosis, to effectively inhibit tumor growth. STAT3 is a critical transcription factor that plays multiple roles in tumor development and malignant progression ${ }^{71}$. Our previous studies demonstrated that STAT3 hyper-activation mediated by the bone marrow X-linked kinase (BMX) is required for maintaining the self-renewal and tumorigenic potential of GSCs in GBM ${ }^{72,73}$. Interestingly, 
GSCs secrete periostin to recruit monocyte-derived TAMs into GBM tumors ${ }^{10}$. In turn, infiltrating TAMs support the maintenance of GSCs through PTN-PTPRZ1 signaling in GBM ${ }^{11}$. Because GSCs play crucial roles in tumor growth and malignant progression, including invasion ${ }^{74,75}$, angiogenesis ${ }^{76,77}$, pericyte generation $^{76}$, blood-tumor barrier (BTB) formation ${ }^{78}$, and therapeutic resistance ${ }^{22,79}$, the reduction of GSCs caused by the decreased pTAMs induced by BACE1 inhibition with MK-8931 may also partially contribute to the suppression of tumor growth in vivo.

Immunotherapy is a promising therapeutic option for cancer treatment, but the majority of solid tumors, including GBM, respond poorly to current immune checkpoint blockade, partially due to the insufficient $T$ cell infiltration, poor delivery of checkpoint inhibitors such as anti-PD1 to tumors, and the development of resistance to the immune stimulation ${ }^{80}$. However, because most malignant tumors contain abundant $\operatorname{TAMs}^{1,2}$, and we have identified an effective approach to enhance macrophage infiltration into tumors by using a low dose of radiation, macrophage-based immunotherapy through BACE1 inhibition with a small molecular modulator such as MK-8931 has several obvious advantages: (1) This therapy reprograming pTAMs into sTAMs not only inhibits TAM tumor-supportive roles but also promote macrophage phagocytosis to engulf cancer cells. The double hit effectively suppresses tumor growth and malignant progression. (2) As current immunotherapy such as CAR-T or anti-PD1 is too expensive for most patients, using a small molecular modulator such as MK-8931 to facilitate TAM-based immunotherapy will provide a much more economic but effective approach to improve the survival of cancer patients; (3) Redirecting pTAMs into sTAMs by MK-8931 should effectively re-modulate the tumor immune microenvironment to overcome resistance to other therapies; (4) The combined low-dose IR and MK-8931 treatment can be broadly used for most malignant tumors containing abundant or relatively fewer TAMs; (5) Because all BACE1 inhibitors, including MK-9831, developed for AD clinical trials display great ability to penetrate the BBB, immunotherapy with a BACE1 inhibitor will overcome the BBB issue, as the BBB negatively impacts other therapies including immune checkpoint inhibitors in malignant brain tumors; and (6) As BACE1 inhibition by MK-8931, AZD3293, E2609, or CNP520 has been shown to be very safe for patients in AD clinical trials ${ }^{32}$, repurposing these BACE1 inhibitors for macrophage-based immunotherapy should be straightforward. We predict that this alternative immunotherapy through BACE1 inhibition has tremendous potential to effectively improve the survival of patients with malignant cancers, including glioblastoma and brain metastases.

\section{Methods}

\section{Cells}

Cells were cultured in a humidified incubator at $37^{\circ} \mathrm{C}$ with $5 \% \mathrm{CO}_{2}$ and atmospheric oxygen. All cells used in this study were consistently confirmed to be free from mycoplasma by using a MycoFluor ${ }^{\text {TM }}$ Mycoplasma Detection Kit (ThermoFisher, M7006). 293FT cells from Clontech (632180) were maintained in DMEM medium supplemented with $10 \%$ (v/v) fetal bovine serum (FBS, ThermoFisher, 10437-036). Human iPSCs from ALSTEM (iPS11) were grown in mTeSR1 medium (StemCell Technologies, 85850). 
Human iPSC-derived monocytes and macrophages were maintained in X-VIVO ${ }^{\mathrm{TM}} 15$ medium (Lonza, 04418Q). Human U937 cells from ATCC (CRL-1593.2 ${ }^{\text {TM}}$ ) were maintained in RPMI 1640 medium with $10 \%$ ( $\mathrm{v} / \mathrm{v}$ ) FBS. Bone-marrow derived macrophages (BMDMs) were derived from mice according to an established protocol ${ }^{81}$ and cultured in RPMI 1640 medium with $10 \%$ (v/v) FBS. Human GSCs (CCF-3264 and CCF-DI315) were derived from human primary GBMs as previously described $72,82,83$ and maintained in Neurobasal medium (Invitrogen, 12349015) supplemented with B-27 (Invitrogen, 12587010), glutamine (2 mM, ThermoFisher, 35050061), non-essential amino acids (ThermoFisher, 11140050), sodium pyruvate (1 mM, ThermoFisher, 11360070), epidermal growth factor (EGF, $20 \mathrm{ng} / \mathrm{mL}$, Goldbio, 1150-04100), and basic fibroblast growth factor (bFGF, $20 \mathrm{ng} / \mathrm{mL}$, R\&D Systems, 4114-TC-01M). Unless otherwise indicated, the Gibco ${ }^{\circledR}$ antibiotic-antimycotic (ThermoFisher, 15240062) was used to prevent contamination in all the media.

\section{Human GBM Surgical Specimens}

Surgical specimens of human GBMs were collected from the Brain Tumor and Neuro-Oncology Center of the Cleveland Clinic according to a protocol approved by the Cleveland Clinic Institutional Review Broad. GBM surgical specimens were used for isolation of GSCs and immunofluorescent analyses. Specifically, the human GBM GSCs CCF-DI315 and CCF-3264 were derived from a 49-year old male patient and a 65year female patient, respectively. CCF-4321 primary GBM specimen was collected from a 74-year old male patient. CCF-DI257 GBM specimen was collected from a 55-year old male patient. CCF-3303 GBM specimen was collected from a 36-year old female patient. CW-1797 GBM specimen was collected from a 57-year old male patient. CW-1798 GBM specimen was collected from a 47-year old male patient. CCF2445 GBM specimen was collected from a 50-year old male patient.

\section{Mice}

All animal experiments were performed in accordance with protocols approved by the IACUC at the Cleveland Clinic Lerner Research Institute. Both male and female NSG mice (NOD.Cg-

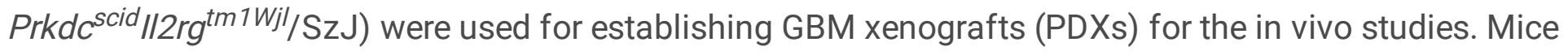
were maintained in a 12-hour light/12-hour dark cycle, and provided with sterilized water and food ad libitum at the Biological Resource Unit of the Cleveland Clinic Lerner Research Institute.

\section{Chemicals and Reagents}

MK-8931 was purchased from Selleckchem (S8173) and Medkoo (331024), dissolved at a concentration of $100 \mathrm{mg} / \mathrm{mL}$ in dimethyl sulfoxide (DMSO, Santa Cruz, sc-358801) as a stock solution, and stored at $-20^{\circ} \mathrm{C}$ until use. D-Luciferin was bought from GoldBio (LUCK-10G), prepared at a concentration of 15 $\mathrm{mg} / \mathrm{mL}$ in sterile water as a stock solution, and stored at $-20^{\circ} \mathrm{C}$ until use. Etoposide (sc-3512B) was from Santa Cruz, prepared at a concentration of $1 \mathrm{mM}$ in DSMO, stored at $-20^{\circ} \mathrm{C}$ until use. $32 \%$ Paraformaldehyde (PFA, 15714) was from Electron Microscopy Sciences and diluted to $4 \%$ with PBS before use. Protease (04693159001) and phosphatase inhibitors (04906837001) tablets were from 
Roche. Recombinant Human SCF (300-07), VEGF (100-20), IL4 (200-04), IL6 (200-06), IL10 (200-10), sIL6R (200-06RC), and TGF 3 (200-21) were from Peprotech. Recombinant Human M-CSF (574806) and IL3 (578006) were from Biolegend. Recombinant Human bFGF was from R\&D Systems (4114-TC-01M). Recombinant Human EGF was from GoldBio (1150-04-100). According to the manufacturer's instructions, all the recombinant proteins were prepared at a 1,000 $\times$ concentration as stock solutions and stored at $-80^{\circ} \mathrm{C}$ until use. All other chemicals and reagents were purchased from Sigma-Aldrich.

\section{Derivation of Monocytes and Macrophages from Human iPS Cells (iPSCs)}

Human iPSC-derived monocytes and macrophages were prepared in accordance with an established protocol ${ }^{11,84}$. To generate $\mathrm{GFP}^{+}$iPSCs, human iPSCs were seeded in a $10-\mathrm{cm}$ tissue culture dish and transduced with GFP through lentiviral infection for 12 hours. Two days post infection, GFP ${ }^{+}$iPSCs were selected and maintained in the mTeSR1 medium (Lonza) for differentiation to monocytes and macrophages. To induce the formation of embryoid bodies (EBs), GFP ${ }^{+}$iPSCs were seeded on an ultralow attachment plate (Costar, 7007) in $100 \mu \mathrm{L}$ of $\mathrm{mTeSR}^{\mathrm{TM}_{1}}$ medium supplemented with BMP4 (50 $\mathrm{ng} / \mathrm{mL}$, Abcam, ab87063), SCF (20 ng/mL, Peprotech, 300-07), Y27632 (50 $\mu \mathrm{M}$, SellckChem, S1049), and VEGF (20 ng/mL, Peprotech, 100-20). The 96-well ultra-low attachment plate was centrifuged at $800 \mathrm{rpm}$ for three minutes and the plate was placed into an incubator and left for four days. At day $2,50 \mu \mathrm{L}$ of culture medium in the well was aspirated and replaced by $50 \mu \mathrm{L}$ of fresh $\mathrm{mTeSR}^{\mathrm{TM}_{1}}$ medium containing the above inducers. To derive monocytes, ten EBs were transferred to each well of a six-well tissue culture plate and cultured in X-VIVO ${ }^{\mathrm{TM}} 15$ medium (Lonza, 04-418Q) supplemented with IL3 $(25 \mathrm{ng} / \mathrm{mL}$, Biolegend, 578006), M-CSF (100 ng/mL, Biolegend, 574806), glutamine (2 mM, ThermoFisher, $35050061)$, and $\beta$-mercaptoethanol $(0.055 \mathrm{M}$, ThermoFisher, 21985023) for two weeks. The medium was changed every five days. Once monocytes were visible in the supernatant of the cultures, non-adherent monocytes were harvested. To produce macrophages, the iPSC-derived monocytes $\left(1.5 \times 10^{5}\right)$ were plated in each well of 6-well plates and cultured in X-VIVO ${ }^{T M} 15$ medium with $\mathrm{M}-\mathrm{CSF}(100 \mathrm{ng} / \mathrm{mL}$, Biolegend, 574806) for six days. To generate M2-like macrophages, the iPSC-derived monocytes were seeded in each well of 6-well plates and primed by treatment with phorbol 12-myristate 13-acetate (PMA, $5 \mathrm{nM})$ for two days to produce M0 macrophages, which were further treated with IL4 $(20 \mathrm{ng} / \mathrm{mL}$, Peprotech, 200-04), IL 10 (20 ng/mL, Peprotech, 200-10), and TGF $\beta$ (20 ng/mL, Peprotech, 200-21) for three days to generate M2-like macrophages.

\section{Preparation of Bone Marrow-derived Monocytes (BMDMs)}

BMDMs were isolated and cultured according to an established protocol ${ }^{81}$. In brief, mouse bone marrow cells were collected by flushing the femurs and tibias with sterile PBS and then treated by red blood cell lysis buffer to remove red blood cells. The cells were re-suspended and cultured in RPMI 1640 medium with $10 \%$ FBS and M-CSF $(100 \mathrm{ng} / \mathrm{mL})$ for seven days to differentiate into BMDMs.

\section{Identification of Potential Drugs to Activate Macrophage Phagocytosis}


To screen for potential small molecules that activate phagocytosis of iPSC-derived macrophages against cancer cells, GFP ${ }^{+}$iPSC-derived macrophages $\left(5 \times 10^{4}\right.$ cells) were seeded in each well of 24-well plates and treated with small molecules from an inhibitor library (SellckChem) or existing drugs that had been used in clinical trials for other diseases for two days. After washing, the cells were maintained in RPMI 1640 medium for two hours. Next, tdTomato-expressing glioma stem cells (GSCs: CCF-3264) were added to each well and co-incubated with the macrophages in RPMI 1640 medium with 10\% FBS for another two hours. After incubation, the co-cultures were washed three times with warm RPMI 1640 medium to remove free cancer cells, and fluorescent and phase images were captured with a fluorescent microscope. Phagocytosis was detected and measured as inclusion bodies of cancer cells (in red) within macrophages (in green) according to a published study ${ }^{39}$.

To detect the MK-8931-activated phagocytosis of bone marrow-derived macrophages (BMDMs) against cancer cells, BMDMs were treated with DMSO (Control) or MK-8931 $(50 \mu \mathrm{g} / \mathrm{mL})$ for two days and prestained with CellTracker ${ }^{\text {TM }}$ Green CMFDA Dye $(1 \mu \mathrm{M}$, ThermoFisher, C2925). After washing, the BMDMs were maintained in RPMI 1640 medium for two hours. Next, tdTomato-expressing CCF-3264 GSCs (2 × $10^{5}$ cells) were added to each well and co-incubated with the labelled BMDMs in RPMI 1640 medium with $10 \%$ FBS for another two hours. After incubation, the co-cultures were washed several times with warm RPMI 1640 medium to remove free cancer cells, and the fluorescent images were captured with a fluorescent microscope.

\section{Plasmids for Overexpression or Knockdown}

To construct the pCDH-tdTomato vector, full-length tdTomato was amplified from the pCDH-EF1-Luc2P2A-tdTomato (Addgene, 72486) vector with the following primers: Forward: 5'-GCT AGC CCA ATC ATT TAA ATA TAA CTT-3', Reverse: 5'-GCG GCC GCT TAC TTG TAC AGC TCG TCC ATG C-3', and then cloned into the pCDH-CMV-MCS-EF1a-Neo vector at the Nhe1 and Not1 sites. The sequence of inserted tdTomato was confirmed by DNA sequencing. The constitutively active STAT3 (STAT3-C-Flag) and pCDH-luciferase constructs were generated in our lab ${ }^{10,73}$. ShRNAs against BACE1 (shBACE1) or a non-targeting sequence (shNT) were purchased from Sigma-Aldrich. The lentivirus packaging vectors (ps-PAX2 and pCl-VSVG) were from Addgene.

\section{Production of Lentiviruses}

Lentiviruses for expression of shRNAs (shBACE1 or shNT) or overexpression of an ectopic protein (STAT3-C) were produced in 293FT cells and prepared as previously described ${ }^{11,72,78,83}$. Briefly, 293FT cells (Clontech, 632180) were co-transduced with targeting plasmids and packaging vectors $\mathrm{pCI-VSVG}$ (Addgene, 1733) and ps-PAX2 (Addgene, 12260) by using calcium phosphate. Four days after transfection, the supernatants were harvested and virus titers were determined as described previously ${ }^{73}$. For infection, cells were treated with lentivirus at a multiplicity of infection (MOI) of 1 .

\section{Generation of Stable Cell Lines}


To generate tdTomato-expressing stable glioma cells, GSCs (CCF-3264) were transduced with tdTomato by means of lentiviral infection for 12 hours. Two days post-infection, the cells were treated with neomycin $(500 \mu \mathrm{g} / \mathrm{mL}$, Santa Cruz, sc-29065A) for seven days to select stable clones. The expression of tdTomato was confirmed by fluorescence microscopy.

To generate luciferase-expressing stable glioma cells, GSCs (CCF-3264 or CCF-DI315) were transduced with firefly luciferase by means of lentiviral infection for 12 hours. Two days post-infection, the cells were treated with puromycin $(2 \mu \mathrm{g} / \mathrm{mL}$, Fisher Scientific, BP2956100) for seven days to select stable clones. The luciferase activity was confirmed by using the Luciferase Assay System (Promega, E1500).

To establish stable U937 cells expressing shBACE1 or shNT, the cells were transduced with shBACE1 or shNT by means of lentiviral infection. Two days post-infection, the cells were treated with puromycin ( 2 $\mu \mathrm{g} / \mathrm{mL}$ ) for seven days to select stable clones. Immunoblotting was used to test the knockdown efficacy of BACE1 in the U937 stable cells.

\section{Derivation of pTAM-like M2 Macrophages from U937 Cells}

U937-derived pTAM-like M2 macrophages were prepared according to an established protocol ${ }^{11,12}$. In brief, cells grown in a 10-cm tissue culture dish were primed with PMA ( $5 \mathrm{nM})$ for two days to produce M0 macrophages, which were further induced by IL4 (20 ng/mL, Peprotech, 200-04), IL 10 ( $20 \mathrm{ng} / \mathrm{mL}$, Peprotech, 200-10), and TGF $\beta$ (20 ng/mL, Peprotech, 200-21) for three days to generate pTAM-like M2 macrophages.

\section{RNA Isolation and RT-PCR}

Total RNA was isolated from cells by using the PureLink ${ }^{T M}$ RNA Kit (ThermoFisher, 12183020) and reverse-transcribed into CDNA with M-MLV reverse transcriptase (Promega, PR-M1701). Real-time PCR (qPCR) was performed on an ABI 7500 Real-Time PCR System (Applied Biosystems) by using the SYBRgreen qPCR Kit (Alkali Scientific, QS2050). Expression values were normalized to GAPDH. Gene-specific primers includes: BACE1 (forward): 5'-GCA GGG CTA CTA CGT GGA GA-3', BACE1 (reverse): 5'-GTA TCC ACC AGG ATG TTG AGC-3'; GAPDH (forward): 5'-AAG GTG AAG GTC GGA GTC AA C-3', GAPDH (reverse) 5'-GGG GTC ATT GAT GGC AAC AAT A-3'.

\section{Establishment of Glioblastoma Xenografts (PDXs) and Drug Treatment in vivo}

To establish xenografts for in vivo studies, intracranial transplantation of GSCs into the brains of NSG mice was performed as described previously ${ }^{10-12,22,83}$. Briefly, GSCs (CCF-3264 or CCF-DI315) expressing luciferase were injected into the right cerebral cortex at a depth of $3.5 \mathrm{~mm}$. For the co-transplantation experiments, human glioma stem cells (GSCs, CCF-DI315) expressing luciferase in combination with or without the monocyte-derived macrophages expressing shBACE1 (shBACE1: \#1 or \#2) or non-targeting control (shNT) were co-injected into NSG mouse brains through intracranial injection (GSCs:Macrophages $=1: 2$ ). In vivo bioluminescent imaging (IVIS) was performed twice per week to monitor the tumor growth, 
using the Spectrum CT Imaging System (PerkinElmer), before and after treatment. For drug treatment, a stock solution of MK-8931 at $100 \mathrm{mg} / \mathrm{mL}$ in DMSO was diluted in $0.5 \%(\mathrm{w} / \mathrm{v})$ methylcellulose (Sigma-

Aldrich, M0512) to $6 \mathrm{mg} / \mathrm{mL}^{34}$. Mice bearing the xenografts were treated with MK-8931 (30 mg/kg) or the control (DMSO) once daily by oral gavage for two weeks or until the humane endpoint was reached. To collect mouse brains bearing tumors, cardiac perfusion with PBS and 4\% PFA (Electron Microscopy Sciences, 15714) was performed. The brains were fixed and sectioned for further immunofluorescent, histochemical and histological analyses.

\section{Irradiation on Intracranial GBM Xenografts (PDXs)}

Irradiation (IR) was performed with a Pantek X-ray irradiator (once per week) at a low dose (2 Gy). To protect the mice and limit the side effects of irradiation, anesthetized mice were covered with a lead plate and only the tumor implantation sites were exposed to the fractioned radiation. Mice were sacrificed at the indicated time points or upon the appearance of neurological signs. Mouse brains bearing the tumors were collected for further analyses through cardiac perfusion with PBS and 4\% PFA (Electron Microscopy Sciences, 15714). Only animals who suffered accidental death (for example, due to infection or intracranial injection) were excluded from the data analysis.

\section{Immunofluorescent Analysis}

Immunofluorescent staining of tumor tissues or cells was performed as described in our previous publications ${ }^{10-12,22,83}$. In brief, tumor sections or cells were fixed with $4 \%$ PFA for ten minutes, washed three times with cold PBS for five minutes each, permeabilized by $0.5 \%(\mathrm{v} / \mathrm{v})$ triton X-100 (Bio-Rad, 1610407) for ten minutes, and blocked with $3 \%(w / v)$ BSA (Sigma-Aldrich, A7906) in PBS for one hour at room temperature. For Ki67 and pSTAT3 staining, antigen retrieval was performed by incubating the sections in boiled antigen retrieval buffer (Vector Laboratories, $\mathrm{H}-3300$ ) for 15 minutes before permeabilization. Primary antibodies were added to the sections or cells and incubated overnight at $4^{\circ} \mathrm{C}$. Primary antibodies used for immunofluorescence in this study were diluted as described below: antiBACE1 (Abcam, ab2077, 1:50; Thermo Fisher Scientific, MA1-177, 1:50), anti-IBA1 (Abcam, ab5076, 1:200; Wako chemicals, 019-19741, 1:200), anti-CD11b (Bio-Rad, MCA711GT, 1:100), anti-FIZZ1 (Abcam, ab39626, 1:100), anti-CD163 (Santa Cruz, sc-33560 and sc-33715, 1:100), anti-ARG1 (BD Biosciences, 610708, 1:300), anti-HLA-DR (Biolegend, 307602, 1:100), anti-CD11c (BD Pharmingen, 558079, 1:100), anti-TRA-1-85 (RD System, MAB3195, 1:300), anti-CD31 (Dako, M082301, 1:100), anti-cleaved caspase 3 (Cell signaling, 9661, 1:100), anti-Ki67 (Abcam, ab15580, 1:100), anti-SOX2 (Bethyl laboratory, A301-739A, 1:200), and anti-pSTAT3 (Tyr705) (Cell signaling, 9131, 1:100). After the incubation of the primary antibodies, the sections or cells were washed three times with cold PBS for five minutes each and then incubated with the secondary antibodies for one hour at room temperature. The secondary antibodies used in this study included Alexa Fluor ${ }^{\circledR} 488$ Donkey Anti-Mouse IgG (Invitrogen, A-21202, 1:200), Alexa Fluor $^{\circledR} 568$ Donkey Anti-Mouse IgG (Invitrogen, A-10037, 1:200), Alexa Fluor ${ }^{\circledR} 488$ Donkey Anti-Rabbit IgG (Invitrogen, A-21206, 1:200), Alexa Fluor ${ }^{\circledR} 568$ Donkey Anti-Rabbit IgG (Invitrogen, A-10042, 1:200), Alexa Fluor $^{\circledR} 488$ Donkey Anti-Goat IgG (Invitrogen, A-11055, 1:200), Alexa Fluor ${ }^{\circledR} 568$ Donkey Anti-Goat IgG 
(Invitrogen, A-11057, 1:200), Alexa Fluor ${ }^{\circledR} 594$ Donkey Anti-rat IgG (Invitrogen, A-21209, 1:200), Alexa Fluor $^{\circledR} 568$ Goat Anti-Armenian Hamster (Abcam, ab175716, 1:200), and Alexa Fluor ${ }^{\circledR} 488$ Goat AntiRabbit (Invitrogen, A-11008, 1:200). After washing three times with cold PBS for five minutes each, the sections or cells were counterstained by DAPI (Cell Signaling, 4083, 1:5000) and sealed with mounting medium (Sigma-Aldrich, F4680). Finally, images were captured by a fluorescence microscopy (Leica DM4000) and further analyzed with ImageJ software (https://imagej.nih.gov/).

\section{Immunoblot Analysis}

Immunoblot analysis was performed as previously described ${ }^{10-12,22,83,85}$. Briefly, cells were lysed with RIPA buffer [50 mM TrisHCl (pH7.4), 150 mM NaCl, 2 mM EDTA, 1\% (v/v) NP-40, 0.1\% (w/v) SDS, protease inhibitor (one tablet per $10 \mathrm{~mL}$ of RIPA buffer, Roche)] for 20 minutes on ice. For the blots of phosphorylated protein, phosphatase inhibitor (one tablet per $10 \mathrm{~mL}$ RIPA buffer, Roche) was used. The cell lysates or conditioned medium (some experiments) were collected and subjected to SDS-PAGE and blotted onto PVDF membranes (ASI, XR730). After blockade with $5 \%(\mathrm{w} / \mathrm{v})$ non-fat milk (RPI, M17200) in TBST, the membranes were incubated with primary antibodies overnight at $4^{\circ} \mathrm{C}$. After the incubation of the first antibodies, the membranes were washed three times with TBST for ten minutes each. Then, the membranes were incubated with the second HRP-linked antibodies in the $5 \%$ milk for one hour at room temperature. The second HRP-linked antibodies were anti-mouse IgG (Cell signaling, 7076, 1:5000), antirabbit IgG (Cell signaling, 7074, 1:5000), and anti-goat IgG (Santa Cruz, sc-2354, 1:5000). After washing three times with TBST for ten minutes each, signals on the membranes were developed in the ECL HRP substrates (Advansta, K-12045) and images were acquired by a molecular imager (Bio-Rad, Universal Hood II) and analyzed by the Image Lab software (Bio-Rad).

\section{Statistical Analysis}

All bar graphs represent mean \pm SEM unless otherwise indicated. For the survival analysis and correlation between gene expressions in glioblastoma patients, the data were provide by TCGA and downloaded from GlioVis. For the survival analysis of glioblastoma patients, the patients were divided into Bace $1^{\text {high }}$ and Bace $1^{\text {low }}$ groups with GlioVis (http://gliovis.bioinfo.cnio.es/) and Kaplan-Meier survival curves were generated. The log-rank survival analysis was performed with GraphPad Prism 5 software (https://www.graphpad.com/) to compare significance among different groups. All quantitative data presented were mean \pm SEM from at least 3 repeats or samples per data point. Experimental details such as number of animals or cells and experimental replication were provided in the figure legends. Data inclusion/exclusion criteria was not applied in this study. Significant differences were determined between two groups using the Student's t test or among multiple groups using one-way ANOVA and statistical significance was set at $p<0.05$.

\section{Declarations}

Acknowledgments 
We thank the Brain Tumor and Neuro-Oncology Centers at the Cleveland Clinic for providing surgical specimens of glioblastoma for this study. We greatly appreciate the help provided by Ms. Mary McGraw from the Brain Tumor Bank at Cleveland Clinic. We thank the Flow Cytometry Core, Imaging Core, and Central Cell Services at Cleveland Clinic Lerner Research Institute for their assistance. This work was supported by Cleveland Clinic Foundation and NIH R01 grants NS091080 and NS099175 (to S.B.). This work utilized an IVIS system (Spectrum CT) that was purchased with NIH SIG grants 1S10RR031536-01 and S100D018205.

\section{Contributions}

S.B. developed the working hypothesis and scientific concept, designed the experimental approaches, oversaw the study, and prepared the manuscript. K.Z. designed the experiments, performed most experiments, analyzed and prepared the data, and wrote the initial draft of manuscript. Z.H. generated the iPSC-derived monocytes and macrophages and established the glioblastoma xenograft model. X.F., W.T. and Q.H. prepared the human glioblastoma samples and isolated the GSCs. X.L., T.A.H. and G.R.S. provided scientific input for the manuscript. G.R.S. also edited the manuscript.

\section{Ethics Declarations}

S.B. and K.Z are listed as inventors in a pending patent application (US 62/896,053) related to this study. Other authors declare no competing interests.

\section{Data availability}

The TCGA database, Rembrandt, Gravendeel, and LeeY database can be downloaded from GlioVis data portal (http://gliovis.bioinfo.cnio.es/). All other data supporting the findings of this study are available from the corresponding author upon reasonable request.

\section{References}

1. Klemm, F., et al. Interrogation of the Microenvironmental Landscape in Brain Tumors Reveals Disease-Specific Alterations of Immune Cells. Cell 181, 1643-1660 e1617 (2020).

2. Guerriero, J.L. Macrophages: The Road Less Traveled, Changing Anticancer Therapy. Trends Mol Med 24, 472-489 (2018).

3. Sorensen, M.D., Dahlrot, R.H., Boldt, H.B., Hansen, S. \& Kristensen, B.W. Tumour-associated microglia/macrophages predict poor prognosis in high-grade gliomas and correlate with an aggressive tumour subtype. Neuropathol Appl Neurobiol 44, 185-206 (2018).

4. Gentles, A.J., et al. The prognostic landscape of genes and infiltrating immune cells across human cancers. Nat Med 21, 938-945 (2015).

5. Lambrechts, D., et al. Phenotype molding of stromal cells in the lung tumor microenvironment. Nat Med 24, 1277-1289 (2018). 
6. Chevrier, S., et al. An Immune Atlas of Clear Cell Renal Cell Carcinoma. Cell 169, 736-749 e718 (2017).

7. Gregoire, H., et al. Targeting Tumor Associated Macrophages to Overcome Conventional Treatment Resistance in Glioblastoma. Front Pharmacol 11, 368 (2020).

8. Qian, B.Z. \& Pollard, J.W. Macrophage Diversity Enhances Tumor Progression and Metastasis. Cell 141, 39-51 (2010).

9. Sica, A., Schioppa, T., Mantovani, A. \& Allavena, P. Tumour-associated macrophages are a distinct M2 polarised population promoting tumour progression: Potential targets of anti-cancer therapy. Eur $\mathrm{J}$ Cancer 42, 717-727 (2006).

10. Zhou, W., et al. Periostin secreted by glioblastoma stem cells recruits M2 tumour-associated macrophages and promotes malignant growth. Nat Cell Bio/ 17, 170-182 (2015).

11. Shi, Y., et al. Tumour-associated macrophages secrete pleiotrophin to promote PTPRZ1 signalling in glioblastoma stem cells for tumour growth. Nat Commun 8, 15080 (2017).

12. Tao, W., et al. Dual Role of WISP1 in maintaining glioma stem cells and tumor-supportive macrophages in glioblastoma. Nat Commun 11, 3015 (2020).

13. Vitale, I., Manic, G., Coussens, L.M., Kroemer, G. \& Galluzzi, L. Macrophages and Metabolism in the Tumor Microenvironment. Cell Metab 30, 36-50 (2019).

14. Grabowski, M.M., et al. Immune suppression in gliomas. J Neurooncol (2020).

15. Zhao, J., et al. Immune and genomic correlates of response to anti-PD-1 immunotherapy in glioblastoma. Nat Med 25, 462-469 (2019).

16. Glass, R. \& Synowitz, M. CNS macrophages and peripheral myeloid cells in brain tumours. Acta Neuropathologica 128, 347-362 (2014).

17. Omuro, A. \& DeAngelis, L.M. Glioblastoma and other malignant gliomas: a clinical review. JAMA 310, 1842-1850 (2013).

18. Zhang, H., et al. Glioblastoma Treatment Modalities besides Surgery. J Cancer 10, 4793-4806 (2019).

19. Reardon, D.A., et al. Effect of Nivolumab vs Bevacizumab in Patients With Recurrent Glioblastoma: The CheckMate 143 Phase 3 Randomized Clinical Trial. Jama Oncol (2020).

20. Wen, P.Y. \& Kesari, S. Malignant gliomas in adults. N Engl J Med 359, 492-507 (2008).

21. Stupp, R., et al. Effects of radiotherapy with concomitant and adjuvant temozolomide versus radiotherapy alone on survival in glioblastoma in a randomised phase III study: 5-year analysis of the EORTC-NCIC trial. Lancet Oncol 10, 459-466 (2009).

22. Bao, S., et al. Glioma stem cells promote radioresistance by preferential activation of the DNA damage response. Nature 444, 756-760 (2006).

23. Prager, B.C., Bhargava, S., Mahadev, V., Hubert, C.G. \& Rich, J.N. Glioblastoma Stem Cells: Driving Resilience through Chaos. Trends Cancer 6, 223-235 (2020). 
24. Lathia, J.D., Heddleston, J.M., Venere, M. \& Rich, J.N. Deadly teamwork: neural cancer stem cells and the tumor microenvironment. Cell Stem Cell 8, 482-485 (2011).

25. Zhou, W. \& Bao, S. Reciprocal Supportive Interplay between Glioblastoma and Tumor-Associated Macrophages. Cancers (Basel) 6, 723-740 (2014).

26. Gordon, S.R., et al. PD-1 expression by tumour-associated macrophages inhibits phagocytosis and tumour immunity. Nature 545, 495-499 (2017).

27. Vassar, R., et al. beta-secretase cleavage of Alzheimer's amyloid precursor protein by the transmembrane aspartic protease BACE. Science 286, 735-741 (1999).

28. Yan, R.Q., et al. Membrane-anchored aspartyl protease with Alzheimer's disease beta-secretase activity. Nature 402, 533-537 (1999).

29. Sinha, S., et al. Purification and cloning of amyloid precursor protein beta-secretase from human brain. Nature 402, 537-540 (1999).

30. Hussain, l., et al. Identification of a novel aspartic protease (Asp 2) as beta-secretase. Mol Cell Neurosci 14, 419-427 (1999).

31. Yan, R.Q. \& Vassar, R. Targeting the beta secretase BACE1 for Alzheimer's disease therapy. Lancet Neurol 13, 319-329 (2014).

32. Panza, F., et al. BACE inhibitors in clinical development for the treatment of Alzheimer's disease. Expert Review of Neurotherapeutics 18, 847-857 (2018).

33. Scott, J.D., et al. Discovery of the 3-Imino-1,2,4-thiadiazinane 1,1-Dioxide Derivative Verubecestat (MK-8931)-A beta-Site Amyloid Precursor Protein Cleaving Enzyme 1 Inhibitor for the Treatment of Alzheimer's Disease. J Med Chem 59, 10435-10450 (2016).

34. Kennedy, M.E., et al. The BACE1 inhibitor verubecestat (MK-8931) reduces CNS beta-amyloid in animal models and in Alzheimer's disease patients. Sci Trans/ Med 8(2016).

35. Forman, M., et al. Safety, Tolerability, and Pharmacokinetics of the beta-Site Amyloid Precursor Protein-Cleaving Enzyme 1 Inhibitor Verubecestat (MK-8931) in Healthy Elderly Male and Female Subjects. Clin Trans/ Sci (2019).

36. Chris Min, K., et al. Pharmacokinetics and Pharmacodynamics of the BACE1 Inhibitor Verubecestat (MK-8931) in Healthy Japanese Adults: A Randomized, Placebo-Controlled Study. Clin Pharmacol Ther 105, 1234-1243 (2019).

37. Egan, M.F., et al. Randomized Trial of Verubecestat for Mild-to-Moderate Alzheimer's Disease. New Engl J Med 378, 1691-1703 (2018).

38. Egan, M.F., et al. Randomized Trial of Verubecestat for Prodromal Alzheimer's Disease. N Engl J Med 380, 1408-1420 (2019).

39. Chen, J., et al. SLAMF7 is critical for phagocytosis of haematopoietic tumour cells via Mac-1 integrin. Nature 544, 493-+ (2017).

40. Yanagimachi, M.D., et al. Robust and highly-efficient differentiation of functional monocytic cells from human pluripotent stem cells under serum- and feeder cell-free conditions. Plos One 8, e59243 
(2013).

41. Thaisrivongs, D.K., et al. Synthesis of Verubecestat, a BACE1 Inhibitor for the Treatment of Alzheimer's Disease. Org Lett 18, 5780-5783 (2016).

42. Pyonteck, S.M., et al. CSF-1R inhibition alters macrophage polarization and blocks glioma progression. Nat Med 19, 1264-+ (2013).

43. Sica, A. \& Mantovani, A. Macrophage plasticity and polarization: in vivo veritas. Journal of Clinical Investigation 122, 787-795 (2012).

44. Murray, P.J. Macrophage Polarization. Annu Rev Physiol 79, 541-566 (2017).

45. Johnson, D.E., O'Keefe, R.A. \& Grandis, J.R. Targeting the IL-6/JAK/STAT3 signalling axis in cancer. Nat Rev Clin Oncol 15, 234-248 (2018).

46. Campbell, I.L., et al. Trans-signaling is a dominant mechanism for the pathogenic actions of interleukin-6 in the brain. J Neurosci 34, 2503-2513 (2014).

47. Hunter, C.A. \& Jones, S.A. IL-6 as a keystone cytokine in health and disease. Nat Immuno/ 16, 448457 (2015).

48. Tsukamoto, H., et al. Soluble IL6R Expressed by Myeloid Cells Reduces Tumor-Specific Th1 Differentiation and Drives Tumor Progression. Cancer Res 77, 2279-2291 (2017).

49. Chen, L., et al. IL-6 influences the polarization of macrophages and the formation and growth of colorectal tumor. Oncotarget 9, 17443-17454 (2018).

50. Fu, X.L., et al. Interleukin 6 induces M2 macrophage differentiation by STAT3 activation that correlates with gastric cancer progression. Cancer Immunol Immunother 66, 1597-1608 (2017).

51. Gaillard, J., et al. Interleukin-6 receptor signaling. II. Bio-availability of interleukin-6 in serum. Eur Cytokine Netw 10, 337-344 (1999).

52. Peters, M., et al. The function of the soluble interleukin 6 (IL-6) receptor in vivo: sensitization of human soluble IL- 6 receptor transgenic mice towards IL- 6 and prolongation of the plasma half-life of IL-6. J Exp Med 183, 1399-1406 (1996).

53. Zegeye, M.M., et al. Activation of the JAK/STAT3 and PI3K/AKT pathways are crucial for IL-6 transsignaling-mediated pro-inflammatory response in human vascular endothelial cells. Cell Commun Signal 16, 55 (2018).

54. Vatner, R.E. \& Formenti, S.C. Myeloid-Derived Cells in Tumors: Effects of Radiation. Semin Radiat Oncol 25, 18-27 (2015).

55. Kioi, M., et al. Inhibition of vasculogenesis, but not angiogenesis, prevents the recurrence of glioblastoma after irradiation in mice. J Clin Invest 120, 694-705 (2010).

56. Hambardzumyan, D., Gutmann, D.H. \& Kettenmann, H. The role of microglia and macrophages in glioma maintenance and progression. Nat Neurosci 19, 20-27 (2016).

57. Razavi, S.M., et al. Immune Evasion Strategies of Glioblastoma. Front Surg 3, 11 (2016).

58. Butowski, N., et al. Orally administered colony stimulating factor 1 receptor inhibitor PLX3397 in recurrent glioblastoma: an Ivy Foundation Early Phase Clinical Trials Consortium phase II study. 
Neuro Oncol 18, 557-564 (2016).

59. Papadopoulos, K.P., et al. First-in-Human Study of AMG 820, a Monoclonal Anti-Colony-Stimulating Factor 1 Receptor Antibody, in Patients with Advanced Solid Tumors. Clin Cancer Res 23, 5703-5710 (2017).

60. Gelderblom, H., et al. Nilotinib in locally advanced pigmented villonodular synovitis: a multicentre, open-label, single-arm, phase 2 trial. Lancet Oncol 19, 639-648 (2018).

61. von Roemeling, C.A., et al. Therapeutic modulation of phagocytosis in glioblastoma can activate both innate and adaptive antitumour immunity. Nat Commun 11, 1508 (2020).

62. Guillon, J., et al. Regulation of senescence escape by TSP1 and CD47 following chemotherapy treatment. Cell Death Dis 10, 199 (2019).

63. Yan, R. Physiological Functions of the beta-Site Amyloid Precursor Protein Cleaving Enzyme 1 and 2. Front Mol Neurosci 10, 97 (2017).

64. Hampel, H., et al. The beta-Secretase BACE1 in Alzheimer's Disease. Biol Psychiatry (2020).

65. Farah, M.H., et al. Reduced BACE1 Activity Enhances Clearance of Myelin Debris and Regeneration of Axons in the Injured Peripheral Nervous System. J Neurosci 31, 5744-5754 (2011).

66. Liu, L.J., et al. Increased TNFR1 expression and signaling in injured peripheral nerves of mice with reduced BACE1 activity. Neurobiol Dis 93, 21-27 (2016).

67. Barker, H.E., Paget, J.T.E., Khan, A.A. \& Harrington, K.J. The tumour microenvironment after radiotherapy: mechanisms of resistance and recurrence. Nat Rev Cancer 15, 409-425 (2015).

68. Ruffell, B. \& Coussens, L.M. Macrophages and therapeutic resistance in cancer. Cancer Cel/ 27, 462472 (2015).

69. Genard, G., Lucas, S. \& Michiels, C. Reprogramming of Tumor-Associated Macrophages with Anticancer Therapies: Radiotherapy versus Chemo- and immunotherapies. Frontiers in Immunology 8(2017).

70. Lee, M., Park, J.J., Ko, Y.G. \& Lee, Y.S. Cleavage of ST6Gal I by radiation-induced BACE1 inhibits golgianchored ST6Gal I-mediated sialylation of integrin beta1 and migration in colon cancer cells. Radiat Oncol 7, 47 (2012).

71. Yu, H., Pardoll, D. \& Jove, R. STATs in cancer inflammation and immunity: a leading role for STAT3. Nat Rev Cancer 9, 798-809 (2009).

72. Shi, Y., et al. Ibrutinib inactivates BMX-STAT3 in glioma stem cells to impair malignant growth and radioresistance. Sci Transl Med 10(2018).

73. Guryanova, O.A., et al. Nonreceptor tyrosine kinase BMX maintains self-renewal and tumorigenic potential of glioblastoma stem cells by activating STAT3. Cancer Cell 19, 498-511 (2011).

74. Krusche, B., et al. EphrinB2 drives perivascular invasion and proliferation of glioblastoma stem-like cells. Elife 5(2016).

75. Cheng, L., et al. Elevated invasive potential of glioblastoma stem cells. Biochem Biophys Res Commun 406, 643-648 (2011). 
76. Cheng, L., et al. Glioblastoma Stem Cells Generate Vascular Pericytes to Support Vessel Function and Tumor Growth. Cell 153, 139-152 (2013).

77. Chiao, M.T., Yang, Y.C., Cheng, W.Y., Shen, C.C. \& Ko, J.L. CD133 + glioblastoma stem-like cells induce vascular mimicry in vivo. Curr Neurovasc Res 8, 210-219 (2011).

78. Zhou, W., et al. Targeting Glioma Stem Cell-Derived Pericytes Disrupts the Blood-Tumor Barrier and Improves Chemotherapeutic Efficacy. Cell Stem Cel/ 21, 591-603 e594 (2017).

79. Osuka, S. \& Van Meir, E.G. Overcoming therapeutic resistance in glioblastoma: the way forward. $J$ Clin Invest 127, 415-426 (2017).

80. Sharma, P., Hu-Lieskovan, S., Wargo, J.A. \& Ribas, A. Primary, Adaptive, and Acquired Resistance to Cancer Immunotherapy. Cel/ 168, 707-723 (2017).

81. Weischenfeldt, J. \& Porse, B. Bone Marrow-Derived Macrophages (BMM): Isolation and Applications. CSH Protoc 2008, pdb prot5080 (2008).

82. Wang, X., et al. Reciprocal Signaling between Glioblastoma Stem Cells and Differentiated Tumor Cells Promotes Malignant Progression. Cell Stem Cell 22, 514-528 e515 (2018).

83. Fang, X., et al. Deubiquitinase USP13 maintains glioblastoma stem cells by antagonizing FBXL14mediated Myc ubiquitination. J Exp Med 214, 245-267 (2017).

84. van Wilgenburg, B., Browne, C., Vowles, J. \& Cowley, S.A. Efficient, long term production of monocytederived macrophages from human pluripotent stem cells under partly-defined and fully-defined conditions. Plos One 8, e71098 (2013).

85. Fang, X., et al. The zinc finger transcription factor ZFX is required for maintaining the tumorigenic potential of glioblastoma stem cells. Stem Cells 32, 2033-2047 (2014).

\section{Figures}


a

iPSC-derived Macrophages

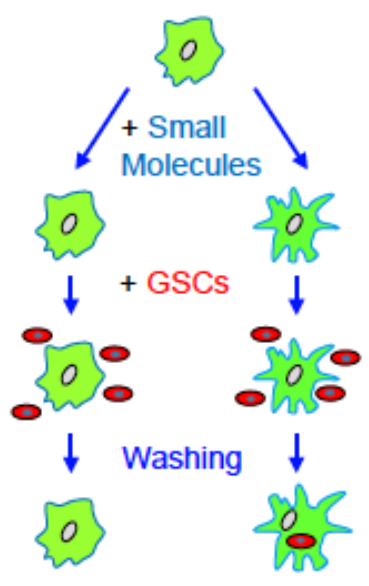

b

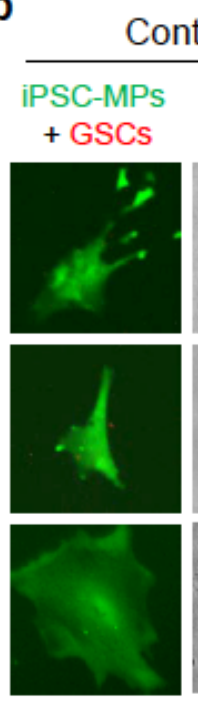

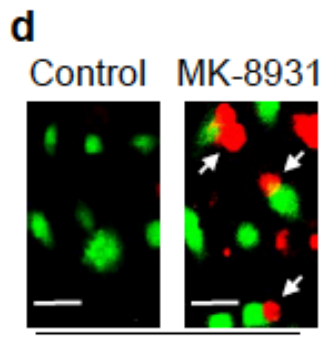

BMDMs + GSCs

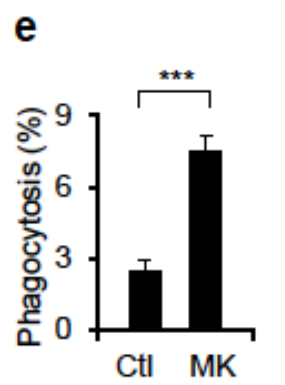

f
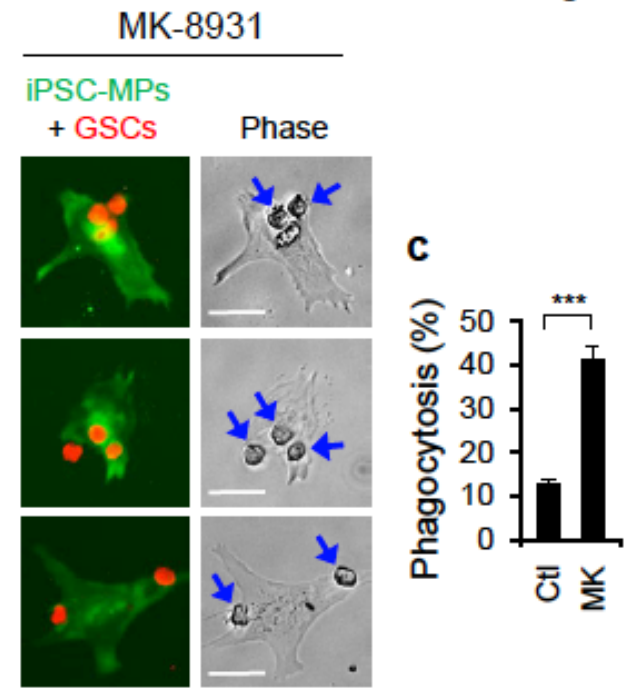

g

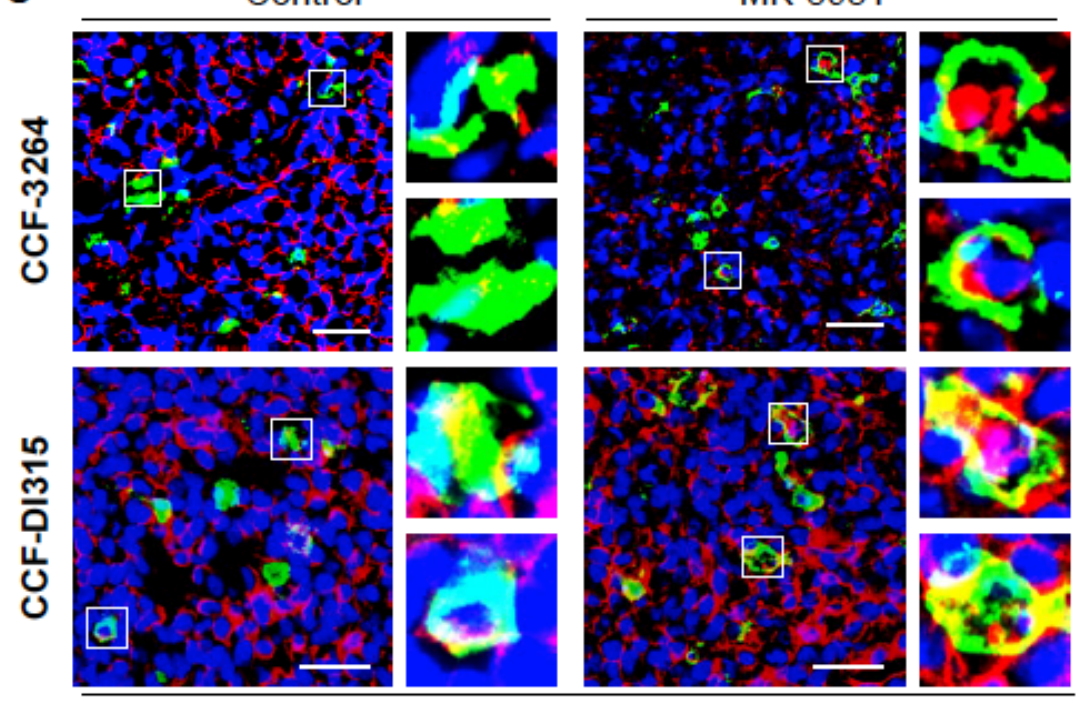

IBA1 TRA-1-85 DAPI h
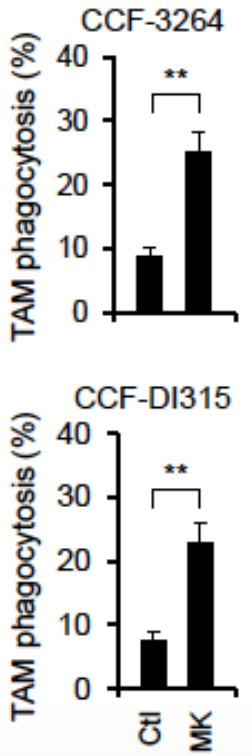

\section{Figure 1}

Identification of the BACE1 Inhibitor MK-8931 as a Potent Activator of Macrophage Phagocytosis of Glioma Cells a, An illustration of the cell-based fluorescent screening assay. Briefly, human iPS cells (iPSC)-derived macrophages (GFP+, in green) were seeded in wells of 24-well plates and treated with different small molecules (in triplicate) for two days. Then, tdTomato+ glioma stem cells (GSCs, in red) were added to each well and co-incubated with the macrophages for two hours. After washing away free 
tumor cells, the images were captured by a fluorescent microscope and then further analyzed. Macrophage phagocytosis was detected as inclusion bodies of GSCs (in red) within macrophages (in green). b,c, In vitro macrophage phagocytosis assay showing that the BACE1 inhibitor MK-8931 promoted phagocytosis of iPSC-derived macrophages (iPSC-MPs) against GSCs. The iPSC-derived macrophages (GFP+) were treated with MK-8931 $(50 \mu \mathrm{g} / \mathrm{mL})$ or the vehicle control for two days and then co-incubated with tdTomato+ GSCs (CCF-3264, in red) to detect macrophage phagocytosis, as illustrated in a. Representative fluorescent and phase contrast images (b) and quantification (c) showing that MK-8931 potently induced phagocytosis of iPSC-derived macrophages (in green) against GSCs (in red). Scale bars

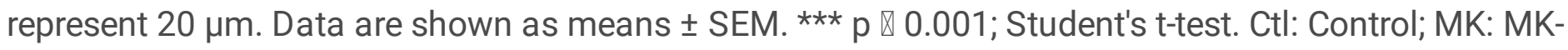
8931. d,e, In vitro macrophage phagocytosis assay showing that MK-8931 also promoted phagocytosis of bone marrow-derived macrophages (BMDMs) against GSCs. BMDMs were treated with MK-8931 (50 $\mu \mathrm{g} / \mathrm{mL}$ ) or the vehicle control for two days and then labelled with green fluorescent dye (CMFDA). The labelled BMDMs (in green) were co-incubated with tdTomato-expressing human GSCs (CCF-3264, in red) for detecting macrophage phagocytosis as illustrated in a. Representative fluorescent images (d) and quantification (e) showing that MK-8931 significantly promoted phagocytosis of BMDMs (in green) against GSCs (in red). Scale bars represent $20 \mu \mathrm{m}$. Data are shown as means \pm SEM. ${ }^{\star \star \star} p \vee 0.001$; Student's t-test. Ctl: Control; MK: MK-8931. f, Immunofluorescent analysis of BACE1 expression in iPSCderived macrophages and the matched iPSCs (control). The iPSC-derived macrophages (GFP+) and the iPSCs (GFP+) were immune-stained with a specific antibody against BACE1 (in red) and counterstained with DAPI (in blue). Representative immunofluorescent images showing that BACE1 is expressed by the iPSC-derived macrophages but not by the iPSCs. Scale bars represent $20 \mu \mathrm{m}$. g,h, In vivo phagocytosis of TAMs against human glioma cells promoted by MK-8931 treatment. Briefly, human GSCs (CCF-3264 or CCF-DI315) were transplanted into NSG mouse brains through intracranial injection to establish GBM xenografts. Seven days after transplantation, the tumor-bearing mice were treated with MK-8931 (30 $\mathrm{mg} / \mathrm{kg}$ ) or the vehicle control once daily by oral gavage for two weeks. After treatment, the GBM xenografts were harvested, and frozen brain sections were immune-stained with specific antibodies against IBA1 (in green, detecting TAMs) and the human cell antigen TRA-1-85 (in red, detecting glioma cells), and counterstained with DAPI (in blue). Representative images (g) showing in vivo TAM phagocytosis as inclusion bodies of glioma cells (TRA-1-85+, in red) within TAMs (IBA1+, in green) in the MK-8931 treated tumors but not in the control tumors. Quantification (h) showing that MK-9831 treatment significantly increased the inclusion bodies of glioma cells (TRA-1-85+, in red) within TAMs (IBA1+, in green), indicating that MK-8931 treatment promoted TAM phagocytosis to engulf glioma cells in GBM tumors. Scale bars represent $30 \mu \mathrm{m}$. Data are shown as means \pm SEM. ** $\mathrm{p} \otimes 0.01$; Student's ttest. Ctl: Control; MK: MK-8931. 
a

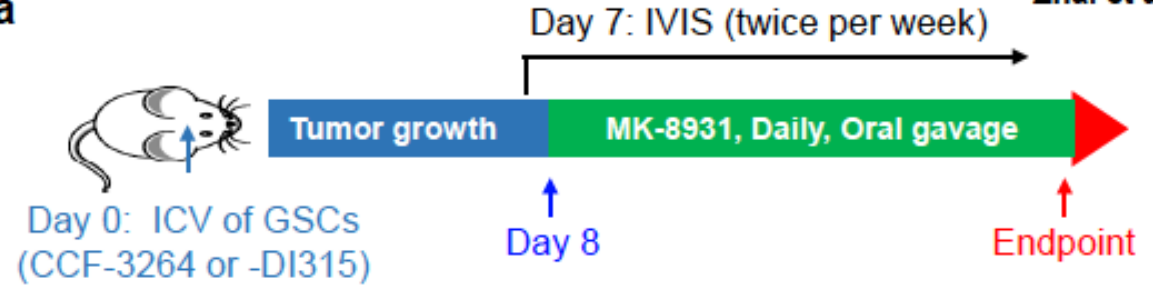

b

GSCs: CCF-3264

GSCs: CCF-DI315
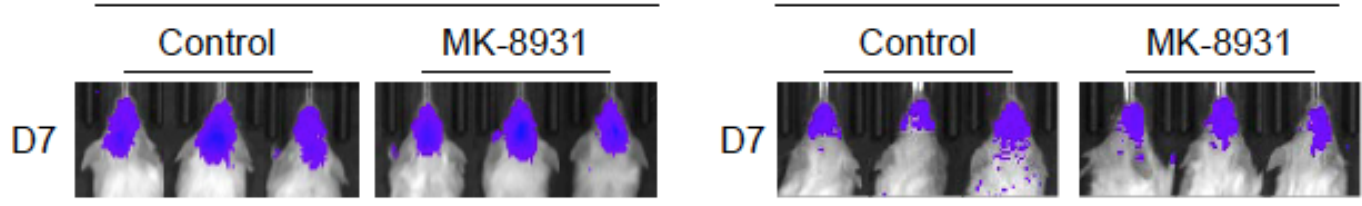

D8: Start treatment
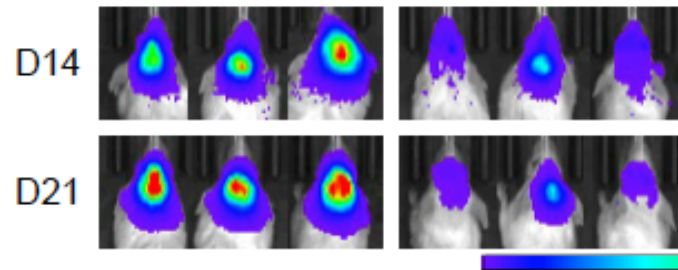

D8: Start treatment

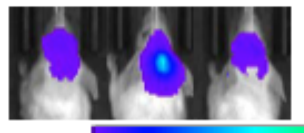

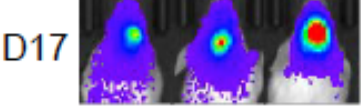
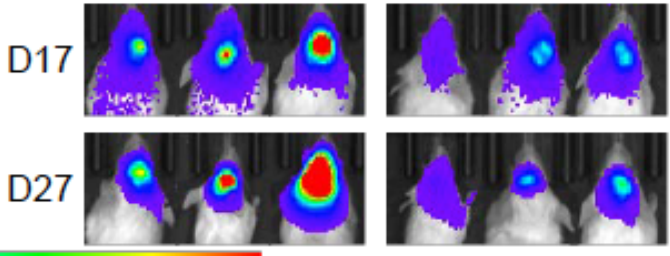

0

60,000

C

GSCs: CCF-3264

GSCs: CCF-DI315
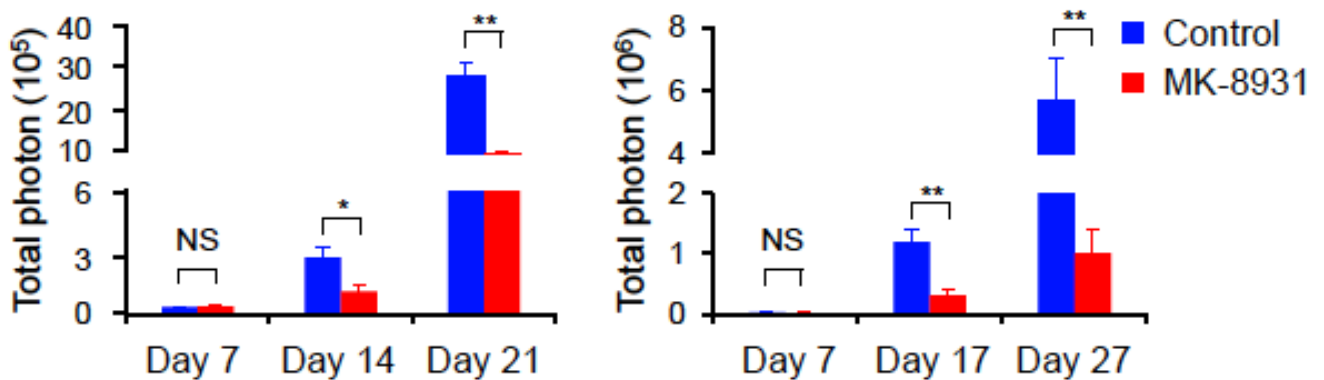

d

GSCs: CCF-3264

GSCs: CCF-DI315
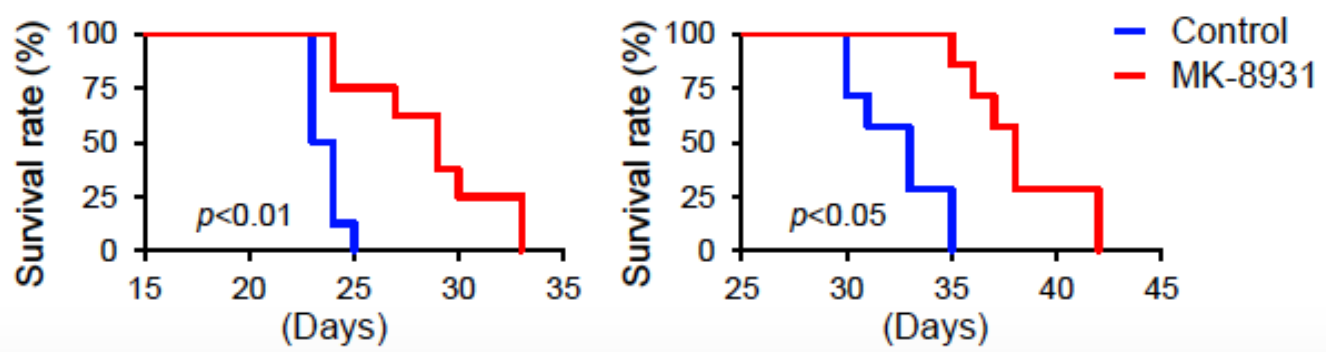

Figure 2

Targeting BACE1 by MK-8931 Potently Inhibits Glioblastoma Growth and Extends Survival of Animals Bearing Intracranial Glioblastoma Xenografts a, A treatment schedule showing the preclinical trial of MK8931 for GBM therapy in GSC-derived xenograft models. Briefly, human glioma stem cells (GSCs: CCF3264 or CCF-DI315) expressing luciferase were transplanted into NSG mouse brains through intracranial injection to establish GBM xenografts. Seven days after transplantation, the tumor-bearing mice were 
treated with MK-8931 (30 mg/kg) or the vehicle control once daily by oral gavage until the appearance of humane endpoints. Bioluminescent imaging (IVIS) were performed twice per week to monitor tumor growth before and after MK-8931 treatment. Mice were maintained until the development of neurological signs to examine the effect of MK-8931 treatment on survival, and brains bearing GBM tumors were harvested for further analyses. b,c, In vivo bioluminescent analysis to monitor intracranial tumor growth of GBM xenografts derived from CCF-3264 or CCF-DI315 GSCs expressing luciferase in mice treated with MK-8931 (30 mg/kg/daily) or the vehicle control. Representative bioluminescent images (b) at the indicated days were shown. Quantification (c) shows the mean bioluminescence of the control mice (7 mice/group) and the MK-8931-treated mice (7 mice/group) on the indicated days. MK-8931 treatment significantly inhibited tumor growth of GBM xenografts. Data are shown as means \pm SEM. ${ }^{*} p<0.05$ and ** $p \otimes 0.01$; Student's t-test. $d$, Kaplan-Meier survival curves of mice bearing GSC-derived GBM xenografts treated with MK-8931 (30 mg/kg/daily) or the vehicle control. Each group contains seven mice. Log-rank analysis was used to assess the significance. MK-8931 treatment significantly extended the survival of mice bearing GBM tumors. $p \otimes 0.05$ and $p \otimes 0.01$ as indicated. 


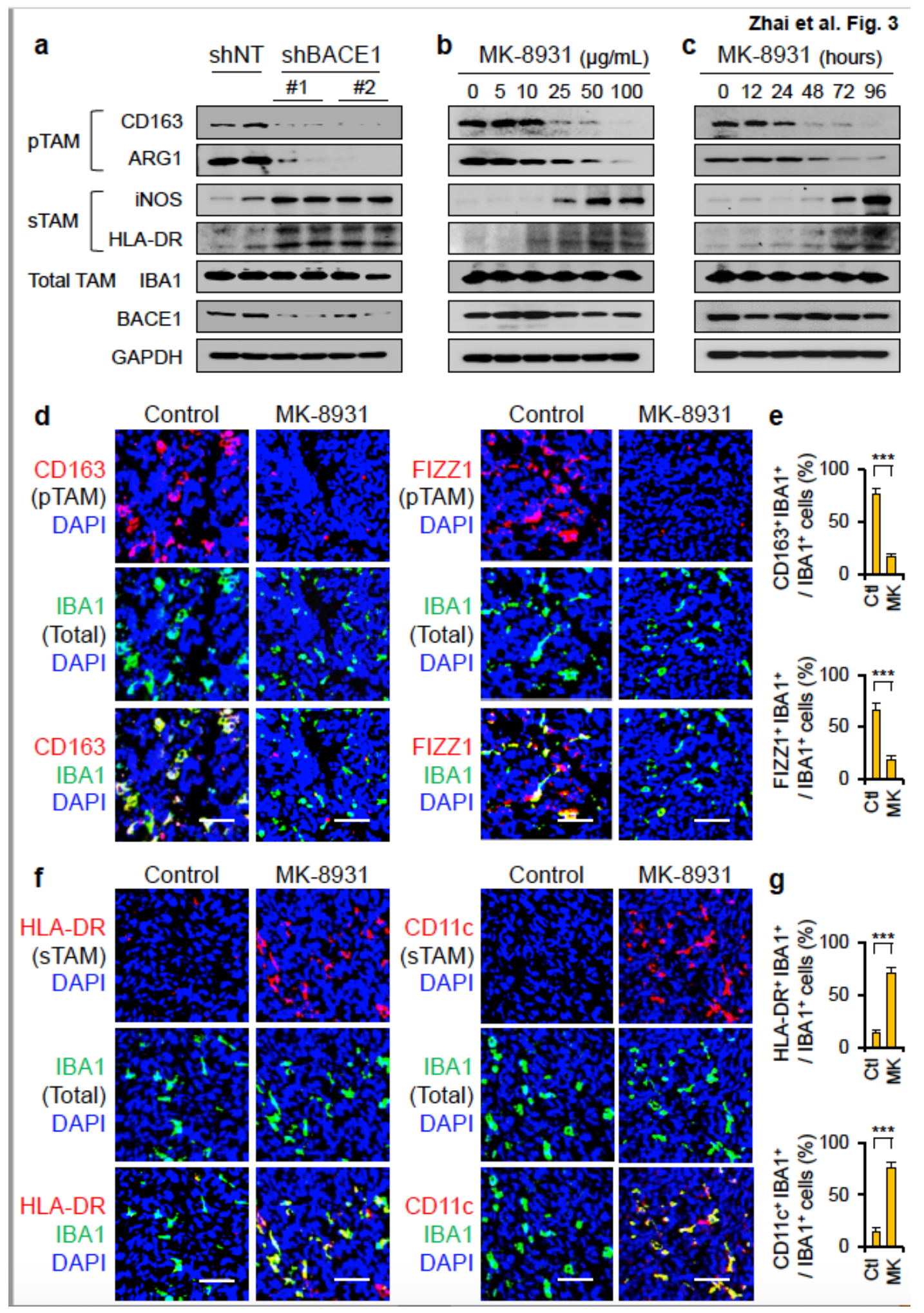

Figure 3

BACE1 Inhibition by MK-8931 Converts Tumor-promoting TAMs (pTAMs) into Tumor-suppressive TAMs (sTAMs) a, Immunoblot analyses of macrophage markers (pTAM: CD163 and ARG1; sTAM: iNOS and HLA-DR; Total TAM: IBA1) in the U937-derived pTAM-like M2 macrophages expressing shRNAs against BACE1 (shBACE1: \#1 or \#2) or non-targeting control (shNT). The U937-derived pTAM-like macrophages were transduced with shBACE1 or shNT through lentiviral infection. BACE1 disruption reduced expression 
of the pTAM markers (CD163 and ARG1) but induced expression of the sTAM markers (iNOS and HLADR). b, Immunoblot analyses of macrophage markers (pTAM: CD163 and ARG1; sTAM: iNOS and HLADR; Total TAM: IBA1) in U937-derived pTAM-like M2 macrophages treated with increased doses of MK$8931(0,5,10,25,50$, and $100 \mu \mathrm{g} / \mathrm{mL})$ for three days. MK-8931 treatment inhibited pTAM marker expression but induced sTAM marker expression in a dose-dependent manner. GAPDH was blotted as the loading control. c, Immunoblot analyses of macrophage markers (pTAM: CD163 and ARG1; sTAM: iNOS and HLA-DR; Total TAM: IBA1) in U937-derived pTAM-like M2 macrophages treated with MK-8931 (50 $\mu \mathrm{g} / \mathrm{mL})$ for different times $(0,12,24,48,72$, or 96 hours). MK-8931 treatment inhibited pTAM marker expression but induced sTAM marker expression in a time-dependent fashion. GAPDH was blotted as the loading control. d,e, Density analysis of pTAMs by immunofluorescent staining of the pTAM marker (CD163 or FIZZ1) and the total TAM marker IBA1 in GBM xenografts treated with MK-8931 or vehicle control. GBM xenografts were established by intracranial transplantation of human GSCs CCF-3264. Seven days after transplantation, the tumor-bearing mice were treated with MK-8931 (30 mg/kg) or the vehicle control once daily by oral gavage for two weeks. Frozen brain tumor sections were immunestained with specific antibodies against CD163 or FIZZ1 (pTAM marker, in red) and IBA1 (total TAM marker, in green) and counterstained with DAPI (in blue). Representative immunofluorescent images (d) showing pTAMs (CD163+ or FIZZ1+, in red) and total TAMs (IBA1+, in green) in the MK-8931-treated or control tumors. Quantifications (e) showing that MK-8931 treatment significantly reduced density of pTAMs (CD163+/IBA1+ or FIZZ1+/IBA1+) in GBM xenografts. Scale bar represents $30 \mu \mathrm{m}$. Data are shown as means \pm SEM. ${ }^{\star \star \star} \mathrm{p} \otimes 0.001$; Student's t-test. $\mathrm{f}, \mathrm{g}$, Density analysis of sTAMs by immunofluorescent staining of the sTAM marker (HLA-DR or CD11c) and the total TAM marker IBA1 in GBM xenografts treated with MK-8931 or a vehicle control. Frozen brain tumor sections were immunestained with specific antibodies against HLA-DR or CD11c (sTAM marker, in red) and IBA1 (total TAM marker, in green) and counterstained with DAPI (in blue). Representative immunofluorescent images (f) showing sTAMs (HLA-DR+ or CD11c+, in red) and total TAMs (IBA1+, in green) in the MK-8931-treated or control tumors. Quantifications (g) showing that MK-8931 treatment significantly increased density of sTAMs (HLA-DR+/IBA1+ or CD11C+/IBA1+) in GBM xenografts. Scale bar represents $30 \mu \mathrm{m}$. Data are shown as means \pm SEM. ${ }^{\star * \star} p \otimes 0.001$; Student's t-test. 


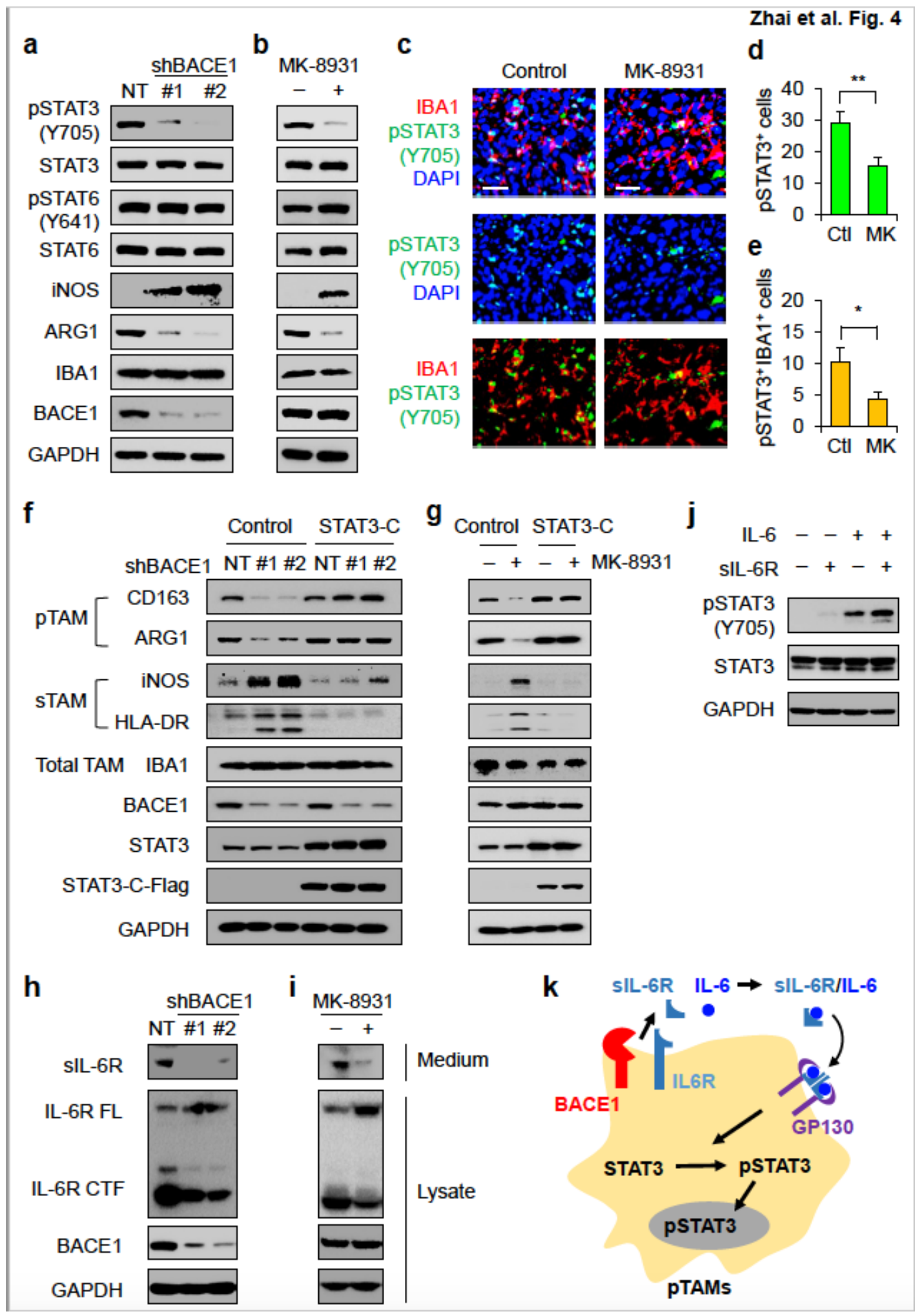

Figure 4

BACE1 Maintains pTAMs by Catalyzing the Shedding IL-6R to Activate Trans IL-6/sIL-6R/STAT3 Signaling a, Immunoblot analyses of STAT3 and STAT6 activation (pSTAT3-Y705 and pSTAT6-Y641) in U937-derived pTAM-like M2 macrophages with or without BACE1 disruption. U937-derived pTAM-like macrophages were transduced with shNT (non-targeting control shRNA) or shBACE1 through lentiviral infection for three days and then harvested for immunoblot analyses of pSTAT3-Y705, STAT3, pSTAT6- 
Y641, STAT6, iNOS (a sTAM marker), ARG1 (a pTAM marker), IBA1 (a total TAM marker), BACE1, and GAPDH (loading control). BACE1 silence reduced STAT3 activating phosphorylation without impact on STAT6 activation in PTAM-like macrophages. b, Immunoblot analyses of STAT3 and STAT6 activation (pSTAT3-Y705 and pSTAT6-Y641) in U937-derived pTAM-like M2 macrophages with or without MK-8931 treatment. U937-derived pTAM-like macrophages were treated with MK-8931 $(50 \mu \mathrm{g} / \mathrm{mL})$ or the vehicle control for three days and then harvested for analyses of pSTAT3-Y705, STAT3, pSTAT6-Y641, STAT6, iNOS (a sTAM marker), ARG1 (a pTAM marker), IBA1 (a total TAM marker), BACE1, and GAPDH (loading control). BACE1 inhibition by MK-8931 reduced STAT3 activating phosphorylation but did not impact STAT6 activation in pTAM-like macrophages. c-e, In vivo analyses of STAT3 activating phosphorylation in TAMs by double immunofluorescent staining of IBA1 and pSTAT3-Y705 in GBM xenografts treated with MK-8931 or a vehicle control. GBM xenografts were established by intracranial transplantation of glioma stem cells (CCF-3264). Seven days after transplantation, the tumor-bearing mice were treated with MK8931 (30 mg/ kg) or the vehicle control once daily by oral gavage for two weeks. Frozen brain tumor sections were immune-stained with specific antibodies against IBA1 (in green) and pSTAT3-Y705 (in red) and counterstained with DAPI (in blue). Representative images (c) showing the fluorescent staining of IBA1 (in green) and the pSTAT3 (in red) in MK-8931-treated or control tumors. Quantification of pSTAT3+ cells (d) shows that MK-8931 treatment significantly reduced STAT3 activating phosphorylation in GBM xenografts. Quantification of IBA1+/pSTAT3+ double positive cells (e) shows that MK-8931 treatment significantly reduced STAT3 activating phosphorylation in TAMs in the tumors. Scale bar represents 30 $\mu \mathrm{m}$. Data are shown as means \pm SEM. ${ }^{*} p<0.05$ and ${ }^{* *} p<0.01$; Student's t-test. Ctl: Control. MK: MK8931. f, Immunoblot analyses of the pTAM markers (CD163 and ARG1) and the sTAM markers (iNOS and HLA-DR) in U937-derived pTAM-like M2 macrophages transduced with the constitutively activated STAT3 (STAT3-C-Flag) or vector (control) in combination with shBACE1 or shNT control. Three days after the transduction, the U937-derived PTAM-like macrophages were harvested for immunoblot analyses of CD163, ARG1, iNOS, HLA-DR, IBA1, BACE1, STAT3, Flag (STAT3-C) and GAPDH (loading control). Ectopic expression of STAT3-C attenuated the PTAM to sTAM switch induced by BACE1 knockdown. $\mathrm{g}$, Immunoblot analyses of the PTAM markers (CD163 and ARG1) and sTAM markers (iNOS and HLA-DR) in U937-derived PTAM-like M2 macrophages transduced with the constitutively activated STAT3 (STAT3-CFlag) or vector (control) in combination with or without MK-8931 treatment. U937-derived pTAM-like macrophages were transduced with STAT3-C-Flag or the vector control and treated with MK-8931 (50 $\mu \mathrm{g} / \mathrm{mL}$ ) or the vehicle control for three days, and then harvested for immunoblot analyses of CD163, ARG1, iNOS, HLA-DR, IBA1, BACE1, STAT3, Flag (STAT3-C) and GAPDH (loading control). Ectopic expression of STAT3-C impaired the PTAM to sTAM switch induced by BACE1 inhibition with MK-8931 treatment. h,i, Immunoblot analyses of the soluble form of IL-6R (sIL-6R), full length IL-6R (FL), C-terminal fragment of IL-6R (CTF), BACE1, and GAPDH in the U937-derived pTAM-like M2 macrophages expressing shBACE1 or shNT (h), or treated with or without MK-8931 (i). BACE1 disruption by shRNA (h) or inhibition by MK-8931 (i) reduced the soluble IL-6R (sIL-6R) in the conditioned medium of macrophages but increased the full length IL-6R (FL) and decreased the C-terminal fragments IL-6R (CTF) in cell lysate of macrophages. j, Immunoblot analyses of pSTAT3-Y705, STAT3, and GAPDH in the U937 macrophages treated with sIL-6R, IL-6, sIL-6R plus IL-6, or the vehicle control. The soluble IL-6R (sIL-6R) enhanced the IL- 
6-mediated STAT3 activation in the U937 macrophages. $k$, A schematic illustration shows that BACE1 maintains pTAMs through the trans-IL-6/sIL-6R/STAT3 signaling. The membrane IL-6R is cleaved by BACE1 to generate the soluble IL-6R (sIL-6R) in pTAMs. Then the sIL-6R captures the IL-6 to form an IL6/sIL-6R complex that binds to gp130 to induce the dimerization of gp130, resulting in increased STAT3 activation to maintain PTAMs.

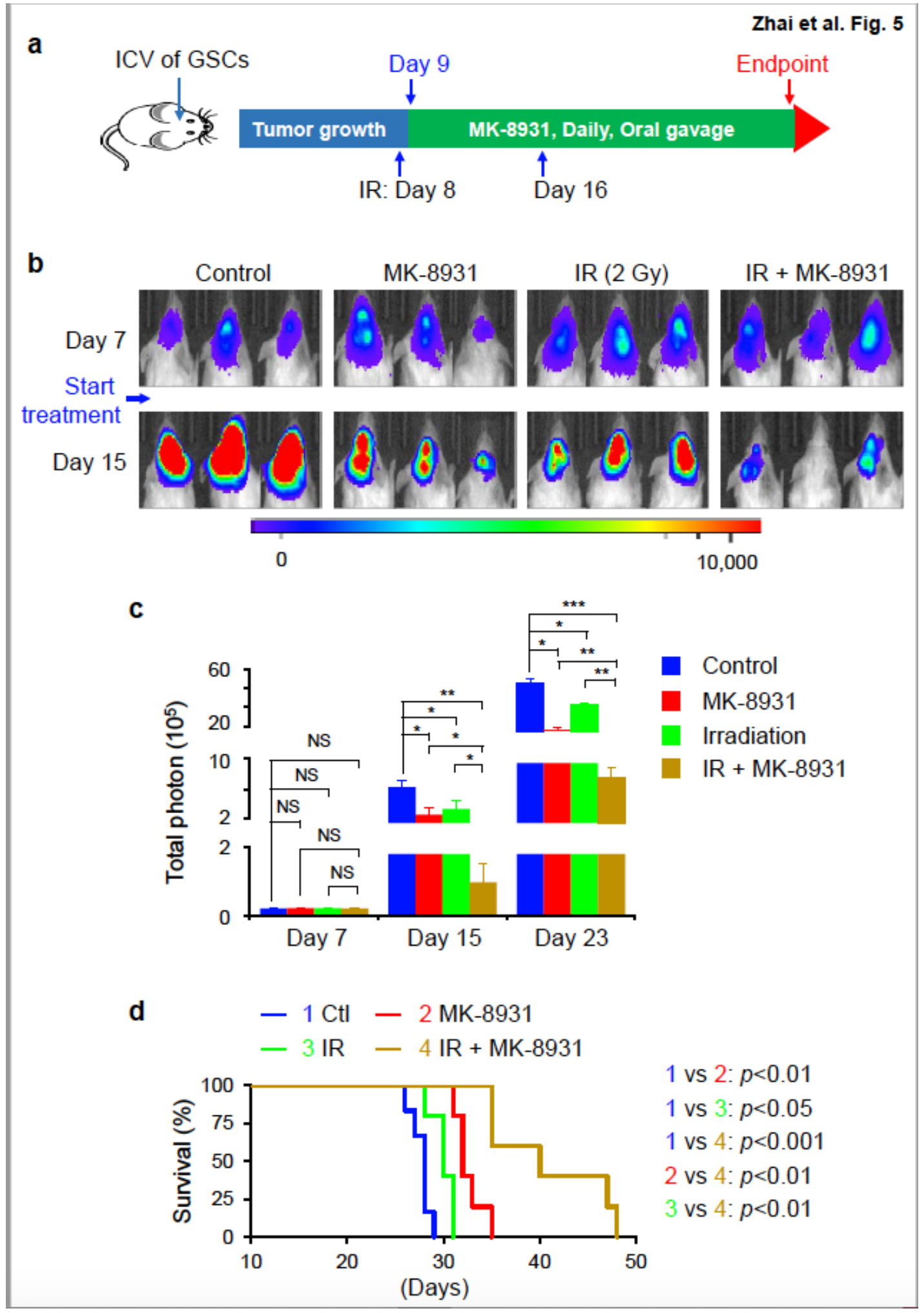

Figure 5 
A Low Dose of Irradiation Synergizes with MK-8931 Treatment to Suppress Glioblastoma Growth and Prolong Survival of Tumor-bearing Mice a, A treatment schedule showing the preclinical trial of MK-8931 treatment in combination with a low dose of irradiation (IR) in GBM xenografts models. Human GSCs CCF-3264 expressing luciferase were transplanted into mouse brains through intracranial injection to establish orthotopic GBM xenografts. Irradiation (2 Gy) was performed on Days 8 and 16. From Day 9, mice were treated with MK-8931 (30 mg/kg) or the vehicle control once daily by oral gavage until the appearance of humane endpoints. Bioluminescent imaging (IVIS) was performed twice per week to monitor tumor growth before and after IR and MK-8931 treatment. b,c, In vivo bioluminescent analysis of tumor growth in mouse brains bearing the GSC-derived GBM xenografts treated with MK-8931, IR, IR plus MK-8931, or the vehicle control. Representative bioluminescent images (b) at Day 7 (before treatment) and Day 15 (treatment for one week) are shown. Quantifications (c) show the mean bioluminescence of the control group, MK-8931-treated group, IR (2 Gy)-treated group, or IR + MK-8931 treated group at Days 7, 15 and 25. Low dose of IR significantly synergized with MK-8931 treatment to inhibit GBM tumor growth. Data are shown as mean \pm SEM. * $p<0.05$, ** $p \otimes 0.01$, $* \star * p \otimes 0.001$, and NS (not significant) $\geq$ 0.05 as indicated; Student's t-test. IR: Irradiation. d, Kaplan-Meier survival curves of the mice bearing the GSC-derived GBM xenografts treated with MK-8931, IR, IR plus MK-8931, or the vehicle control. Low dose of IR synergized with MK-8931 treatment to extend survival of the tumor-bearing animals. Data are shown as mean \pm SEM. Log-rank analysis was used to assess the significance. Significances were shown as indicated. Ctl: Control; IR: Irradiation. 


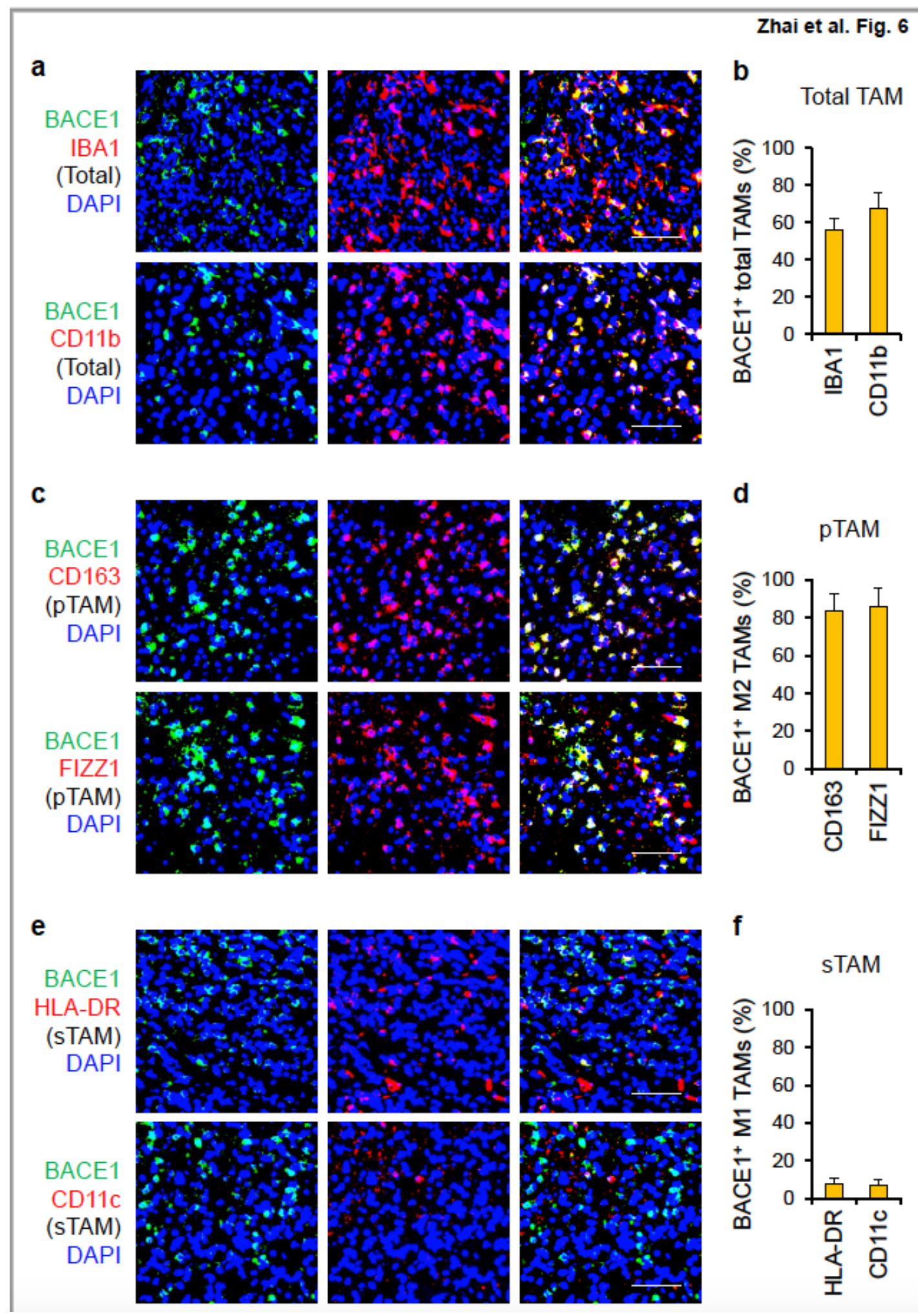

Figure 6

BACE1 Is Preferentially Expressed by pTAMs in Human Primary Glioblastomas a,b, Immunofluorescent analysis of BACE1 and the total TAM marker IBA1 or CD11b in human GBM tumor samples. Frozen sections of GBMs were co-stained with specific antibodies against BACE1 (in green) and IBA1 or CD11b (in red) and then counterstained with DAPI (in blue). Representative immunofluorescent images (a) show the distribution and co-localization of BACE1 (in green) with the total TAM marker IBA1 or CD11b (in red) 
in a human glioblastoma (CCF4321). Quantification (b) shows the fractions of BACE1+ TAMs (BACE1+/IBA1+ or BACE1+/CD11b+ cells) in total TAMs (IBA1+ or CD11b+ cells) in the GBM. c,d, Immunofluorescent analysis of BACE1 and the PTAM marker CD163 or FIZZ1 in human GBMs. Frozen sections of GBMs were co-stained with specific antibodies against BACE1 (in green) and CD163 or FIZZ1 (in red) and then counterstained with DAPI (in blue). Representative immunofluorescent images (c) show the distribution and co-localization of BACE1 (in green) with the pTAM marker CD163 or FIZZ1 (in red) in a GBM (CCF4321). Quantification (d) shows the fractions of BACE1+ pTAMs (BACE1+/CD163+ or BACE1+/FIZZ1+ cells) in pTAMs (CD163+ or FIZZ1+ cells) in the GBM tumor. e,f, Immunofluorescent analysis of BACE1 and the sTAM marker HLA-DR or CD11c in human GBMs. Frozen sections of GBMs were co-stained with specific antibodies against BACE1 (in green) and HLA-DR or CD11c (in red) and then counterstained with DAPI (in blue). Representative immunofluorescent images (e) show the expression of BACE1 (in green) and sTAM markers (HLA-DR or CD11c, in red) in a GBM (CCF4321). Quantification (F) shows the fractions of BACE1+ sTAMs (BACE1+HLA-DR+ or BACE1+CD11c+ cells) in sTAMs (HLA-DR+ or $\mathrm{CD} 11 \mathrm{c}+$ cells) in the GBM. Data are shown as means \pm SEM. Scale bars represent $30 \mu \mathrm{m}$. 


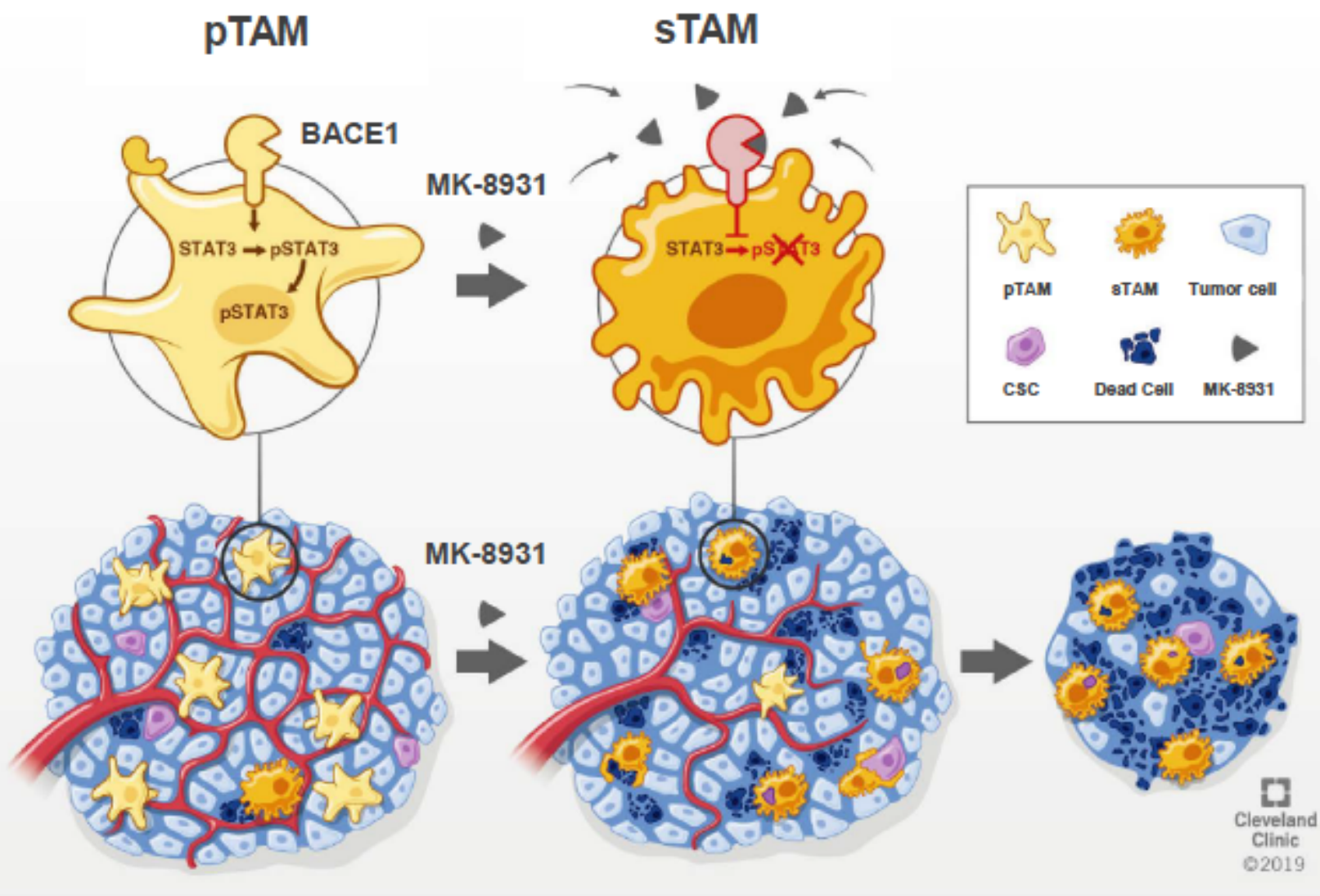

Figure 7

A Schematic Illustration Shows that BACE1 Inhibition by MK-8931 Reprograms pTAMs into sTAMs to Promote TAM Phagocytosis and Suppress Tumor Growth Most malignant tumors including glioblastoma contain abundant tumor-promoting TAMs (pTAMs). BACE1-mediated STAT3 activation is required for maintaining pTAMs. Targeting BACE1 by its inhibitor MK-8931 redirects pTAMs into sTAMs to engulf glioma cells and re-modulate the tumor microenvironment. Thus, BACE1 inhibition by MK-8931 potently suppresses malignant growth of glioblastoma, highlighting the promising therapeutic potential of the macrophage-based immunotherapy through BACE1 inhibition with MK-8931 to improve survival of patients with malignant tumors including glioblastoma 


\section{Supplementary Files}

This is a list of supplementary files associated with this preprint. Click to download.

- SupplementaryFigureswithlegends.pdf 